\title{
Randomized maximum likelihood based posterior sampling
}

\author{
Yuming $\mathrm{Ba}^{1,2}$ - Jana de Wiljes ${ }^{3} \cdot$ Dean S. Oliver ${ }^{4}$ (D) Sebastian Reich ${ }^{3}$
}

Received: 10 January 2021 / Accepted: 27 September 2021 / Published online: 20 December 2021

(C) The Author(s) 2021

\begin{abstract}
Minimization of a stochastic cost function is commonly used for approximate sampling in high-dimensional Bayesian inverse problems with Gaussian prior distributions and multimodal posterior distributions. The density of the samples generated by minimization is not the desired target density, unless the observation operator is linear, but the distribution of samples is useful as a proposal density for importance sampling or for Markov chain Monte Carlo methods. In this paper, we focus on applications to sampling from multimodal posterior distributions in high dimensions. We first show that sampling from multimodal distributions is improved by computing all critical points instead of only minimizers of the objective function. For applications to high-dimensional geoscience inverse problems, we demonstrate an efficient approximate weighting that uses a low-rank Gauss-Newton approximation of the determinant of the Jacobian. The method is applied to two toy problems with known posterior distributions and a Darcy flow problem with multiple modes in the posterior.
\end{abstract}

Keywords Randomized maximum likelihood $\cdot$ Importance sampling $\cdot$ Minimization $\cdot$ Multimodal posterior · Bayesian inverse problem

\section{Introduction}

In several fields, including groundwater management, groundwater remediation, and petroleum reservoir management, there is a need to characterize permeable rock bodies

Dean S. Oliver

deol@ norceresearch.no

Yuming Ba

yumingb9630@163.com

Jana de Wiljes

wiljes@uni-potsdam.de

Sebastian Reich

sebastian.reich@uni-potsdam.de

1 School of Mathematics, Hunan University, Changsha 410082, China

2 Present address: School of Mathematics and Systems Science, Guangdong Polytechnic Normal University, Guangzhou 510665, China

3 Universität Potsdam, Institut für Mathematik, Karl-Liebknecht-Str. 24/25, D-14476 Potsdam, Germany

4 NORCE Norwegian Research Centre, Bergen, Norway whose properties are spatially variable. In most cases, the reservoirs are deeply buried, the number of parameters needed to characterize the porous medium is large and the observations are sparse and indirect [33]. In these applications, the problem of estimating model parameters is almost always underdetermined and the desired solution is not simply a best estimate, but rather a probability density on model parameters conditioned to the observations and to the prior knowledge [39]. Because the model dimension in geoscience applications is always large, the posterior distribution is often represented empirically by samples from the posterior distribution.

Unfortunately, the posterior probability density for reservoir properties, conditional to rate and pressure observations, is typically complex and not easily sampled. Several authors have shown that the log-posterior for subsurface flow problems is not convex in some situations $[34,40,46]$ so that Gaussian approximations of the posterior distribution appear to be dangerous. Markov chain Monte Carlo methods (MCMC) are often considered to provide the gold standard for sampling from the posterior. It is frequently suggested to be the method against which other methods are compared [23], yet it can be difficult to design efficient transition kernels [11,13] and convergence of MCMC can be difficult to assess [17]. The number of 
likelihood function evaluations required to obtain a modest number of independent samples may be excessive for highly nonlinear flow problems [35].

Importance sampling methods can also be considered to be exact sampling methods as they implement Bayes rule directly. They are very difficult to apply in high dimensions, however, as an efficient implementation requires a proposal density that is a good approximation of the posterior [5, 28]. Various methods have been developed in the data assimilation community to ensure that particles are located in regions of high probability density [24]. Although not introduced as importance sampling approaches, a variety of methods based on minimization of a stochastic objective function have been developed, beginning with Kitanidis [21] and Oliver et al. [36] who introduced minimization of a stochastic objective function as a way of simulating samples from an approximation of the posterior when the prior distribution is Gaussian and the errors in observations are Gaussian and additive. The distribution of samples based on minimizers of the objective function was shown to be correct for Gauss-linear problems, but when the observation operator $g(m)$ is nonlinear, it was necessary to weight the samples because the sampling was only approximate in that case.

The randomized maximum likelihood (RML) method approach to sampling has been used without weighting in high dimensional inverse problems with Gaussian priors $[7,9,14,16]$. Weights are seldom computed for several reasons: computation of exact weights is infeasible in large dimensions because the computation of weights requires the second derivative of the observation operator, the proposal density does not always cover the target density, and sampling without weighting sometimes provides a good approximation of the posterior even in posterior distributions with many modes [32]. In practice, the most popular implementations are the ensemble-Kalman based forms of the RML method [10, 20, 38, 45] in which a single average sensitivity is used for minimization so that weighting of samples is not possible.

Bardsley et al. [3] proposed another minimization-based sampling methodology, randomize-then optimize (RTO), in which the need for computation of the second derivative of the observation operator is avoided for weighting. The RTO method has then been modified to allow application in very high dimensions [4], but the method is restricted to posterior distributions with a single mode. Wang et al. [44] discussed the relationship between the cost function in the RML method and the cost function in the RTO method, and showed that the methods are equivalent for linear observation operators. Wang et al. also showed that some approximations to the weights in RML could be computed in high dimensions and provided a useful comparison of sampling distributions.
For nonconvex log-posteriors, there could be many (local) minimizers of the RML and RTO cost functions. Oliver et al. [36] and Oliver [32] suggested that only the global minimizer should be used for sampling, although finding the global minimizer would be difficult to ensure. To improve the likelihood of converging to the global minimizer, they suggested using the unconditional sample from the prior as the starting point for minimization. In contrast, Wang et al. [44] investigated the effect of various strategies for choosing the initial guess on sample distribution and found that a random initial guess worked well.

Unlike previous methods that compute minimizers only, we show that exact sampling is possible when all critical points of a stochastic objective function are computed and properly weighted. Computing the weights accurately, however, for all critical points in high dimensions does not appear to be feasible, but we demonstrate that GaussNewton approximations of the weights provide good approximations for minimizers of the objective function in problems with multimodal posteriors. The Gauss-Newton approximations of weights can be obtained as by-products of Gauss-Newton minimization of the objective function, or as low-rank approximations using stochastic sampling approaches. We also show that valid sampling can be performed without computing all critical points, but by instead randomly sampling of the critical points.

We investigated the performance of both exact sampling and approximate sampling on two small toy problems for which the sampled distribution can be compared with the exact posterior probability density. For a problem with two modes in the posterior pdf, the distribution of samples from weighted RML using all critical points appears to be correct. Approximate sampling using Gauss-Newton approximation of weights and minimizers samples well from both modes, but under-samples the region between modes. The data misfit is not a useful approximation of the weights in this case.

We also applied the approximate sampling method to the problem of estimating permeability in a $2 \mathrm{D}$ porous medium from 25 measurements of pressure. In this case, the distribution of weights was relatively large, even when the log-permeability was distributed as multivariate normal. High-dimensional state spaces such as considered here have a severe effect on importance sampling and remedies such as tempering are suggested to reduce the impact of the dimensionality on the estimation [6].

\section{RML sampling algorithm}

Given a prior Gaussian distribution $\mathrm{N}\left(\bar{m}, C_{M}\right)$ on a set of model parameters $m \in \mathbb{R}^{N_{m}}$ and observations $d^{\mathrm{o}} \in \mathbb{R}^{N_{d}}$ 
which are related to the model parameters through a forward map $g: \mathbb{R}^{N_{m}} \rightarrow \mathbb{R}^{N_{d}}$ for unknown $m^{*}$ and unknown measurement errors $\epsilon \sim \mathrm{N}\left(0, C_{D}\right)$, i.e.,

$d^{\mathrm{o}}=g\left(m^{*}\right)+\epsilon$,

we wish to generate samples $m_{i}, i=1, \ldots, N_{e}$, from the posterior distribution

$\pi_{M}\left(m \mid d^{\mathrm{o}}\right)=\frac{\pi_{M D}\left(m, d^{\mathrm{o}}\right)}{\pi_{D}\left(d^{\mathrm{o}}\right)} \propto \exp (-L(m))$

with negative log likelihood function

$$
\begin{aligned}
L(m)= & \frac{1}{2}(m-\bar{m})^{T} C_{M}^{-1}(m-\bar{m}) \\
& +\frac{1}{2}\left(g(m)-d^{\mathrm{o}}\right)^{T} C_{D}^{-1}\left(g(m)-d^{\mathrm{o}}\right) .
\end{aligned}
$$

The normalisation constant $\pi_{D}\left(d^{\circ}\right)$ is unknown, in general. We will use $\pi_{M}(m):=\pi_{M}\left(m \mid d^{\mathrm{o}}\right)$ in order to simplify notation. A table of nomenclature is included in the Appendix.

In this paper, we will show how to use the RML method in order to produce independent weighted Monte Carlo samples from the posterior distribution Eq. 2. Posterior sampling problems of the form Eq. 2 with negative $\log$ likelihood function Eq. 3 arise from many practical Bayesian inference problems. In practical applications, where the number of model parameters is typically large, the computation of exact weights is infeasible. For those cases we suggest approximations.

\subsection{The trial distribution: RML as proposal step}

The RML method draws samples $\left(m_{i}^{\prime}, \delta_{i}^{\prime}\right), i=1, \ldots, N_{s}$, from the Gaussian distribution

$$
\begin{aligned}
q_{M^{\prime} \Delta^{\prime}}\left(m^{\prime}, \delta^{\prime}\right)= & \frac{1}{(2 \pi)^{N_{m} N_{d} / 2}\left|C_{M}\right|^{1 / 2}\left|C_{D}\right|^{1 / 2}} \\
& \times \exp \left(-\frac{1}{2}\left(m^{\prime}-\bar{m}\right)^{T} C_{M}^{-1}\left(m^{\prime}-\bar{m}\right)\right. \\
& \left.-\frac{1}{2}\left(\delta^{\prime}-d^{\mathrm{o}}\right)^{T} C_{D}^{-1}\left(\delta^{\prime}-d^{\mathrm{o}}\right)\right)
\end{aligned}
$$

for given $\bar{m}$ and $d^{\mathrm{o}}$ and then computes critical points of the cost functional

$$
\begin{aligned}
L_{i}(m)= & \frac{1}{2}\left(m-m_{i}^{\prime}\right)^{T} C_{M}^{-1}\left(m-m_{i}^{\prime}\right) \\
& +\frac{1}{2}\left(g(m)-\delta_{i}^{\prime}\right)^{T} C_{D}^{-1}\left(g(m)-\delta_{i}^{\prime}\right) .
\end{aligned}
$$

by solving

$\nabla_{m} L_{i}(m)=0$,

for $m$. Dropping the subscript $i$, this leads to a map from $(m, \delta)$ to $\left(m^{\prime}, \delta^{\prime}\right)$ defined by

$\left\{\begin{array}{l}m^{\prime}=m+C_{M} G^{T} C_{D}^{-1}(g(m)-\delta) \\ \delta^{\prime}=\delta\end{array}\right.$ which we denote compactly as

$z^{\prime}=\Psi(z)$

where $z=(m, \delta), z^{\prime}=\left(m^{\prime}, \delta^{\prime}\right)$ and the differential of $g$ is denoted $G=D g(m)$. The mapping (Eq. 8) is, in general, not invertible and, hence, a single draw $\left(m_{i}^{\prime}, \delta_{i}^{\prime}\right)$ from Eq. 4 can lead to multiple critical points $\left(m_{j}, \delta_{j}\right) .{ }^{1}$ We therefore introduce the set-valued

$\mathcal{M}_{z^{\prime}}=\Psi^{-1}\left(z^{\prime}\right)$

and denote its elements by $z_{j}\left(z^{\prime}\right) \in \mathcal{M}_{z^{\prime}}, j=1, \ldots, n\left(z^{\prime}\right)$, where $n\left(z^{\prime}\right)$ denotes the cardinality of $\mathcal{M}_{z^{\prime}}$. Each $z$ leads to a unique $z^{\prime}$, hence the sets $\mathcal{M}_{z^{\prime}}$ are disjoint. Let us denote the set of all $z^{\prime}$ for which $n\left(z^{\prime}\right)>0$ by $\mathcal{U}$ and let us assume for now that $\mathcal{U}$ agrees with the support of the distribution $q_{M^{\prime} \Delta^{\prime}}{ }^{2}$

A distribution $q\left(z^{\prime}\right)$ transforms under a map (Eq. 8) into a distribution $p(z)$ according to

$q\left(z^{\prime}\right)=\sum_{z_{i} \in \mathcal{M}_{z^{\prime}}} \frac{p\left(z_{i}\right)}{J\left(z_{i}\right)}$

with Jacobian $J(z)=\operatorname{det}(D \Psi(z))$. We will frequently use the abbreviation $|A|$ for the determinant $\operatorname{det}(A)$ of a matrix $A$. An explicit expression for $p(z)$ is obtained via

$p(z)=n\left(z^{\prime}\right)^{-1} J(z) q\left(z^{\prime}\right)$

for all $z \in \mathcal{M}_{z^{\prime}}$, which satisfies Eq. 9. In the original notation and employing Eq. 7, the transformed distribution $p_{M \Delta}$ is given by

$$
\begin{aligned}
p_{M \Delta}(m, \delta) & :=n\left(m^{\prime}\right)^{-1} q_{M^{\prime} \Delta^{\prime}}\left(m^{\prime}, \delta^{\prime}\right) J(m, \delta) \\
& =n\left(m^{\prime}\right)^{-1} q_{M^{\prime}}\left(m+C_{M} G^{T} C_{D}^{-1}(g(m)-\delta)\right) \\
& \times q_{\Delta^{\prime}}(\delta) J(m, \delta) .
\end{aligned}
$$

Here $n\left(m^{\prime}\right)$ is the total number of critical points of Eq. 5 for each $\left(m^{\prime}, \delta^{\prime}\right)$ and $J(m, \delta)$ denotes the Jacobian determinant associated with the map $(m, \delta) \rightarrow\left(m^{\prime}, \delta^{\prime}\right)$. In the following, we assume that $J \neq 0$ everywhere, i.e., the map is locally invertible. The Jacobian matrix is provided by

$$
\left(\begin{array}{cc}
I+D b(m, \delta) & -C_{M} G^{\mathrm{T}} C_{D}^{-1} \\
0 & I
\end{array}\right)
$$

with $b(m, \delta)=C_{M} G^{\mathrm{T}} C_{D}^{-1}(g(m)-\delta)$.

Hence, given samples, $\left(m_{i}^{\prime}, \delta_{i}^{\prime}\right)$, from Eq. 4 we can easily produce samples, $\left(m_{k}, \delta_{k}\right)$, from the distribution $p_{M \Delta}(m, \delta)$ and would like to use them as importance samples from

\footnotetext{
$\overline{{ }^{1} \text { See Section } 3} .1$ for a sampling problem with a quadratic observation operator, $g$, resulting in a non-invertible mapping. In that example, Eq. 7 is cubic in the variable $m$. Non-invertible mappings appear to be common for Darcy flow problems (e.g., Section 3.3.3).

${ }^{2}$ If there are points $z^{\prime}$ for which $\mathcal{M}_{z^{\prime}}$ is the empty set, that is, $n\left(z^{\prime}\right)=0$, we adjust the PDF $q_{M^{\prime} \Delta^{\prime}}$ such that $q_{M^{\prime} \Delta^{\prime}}\left(z^{\prime}\right)=0$ for $\mathcal{M}_{z^{\prime}}=\emptyset$.
} 
the target distribution $\pi_{M}(m):=\pi_{M}\left(m \mid d^{\mathrm{o}}\right)$ as defined by Eq. 2. Note that the target density $\pi_{M}(m)$ does not specify a distribution in $\delta$ and we will explore this freedom in the subsequent discussion in order to define an efficient importance sampling procedure.

Indeed, we may introduce an extended target distribution by

$\pi_{M \Delta}(m, \delta):=\pi_{M}(m) \pi_{\Delta}(\delta \mid m)$

without changing the marginal distribution in $m$. The conditional distribution $\pi_{\Delta}(\delta \mid m)$ will be chosen to make the proposal density similar to the target density, i.e.,

$\pi_{M \Delta}(m, \delta) \approx p_{M \Delta}(m, \delta)$.

We will find that equality can be achieved for linear forward maps, $g(m)=G m$. In all other cases, samples, $\left(m_{k}, \delta_{k}\right)$ from $p_{M \Delta}(m, \delta)$ will receive an importance weight

$w_{k} \propto \frac{\pi_{M \Delta}\left(m_{k}, \delta_{k}\right)}{p_{M \Delta}\left(m_{k}, \delta_{k}\right)}$

subject to the constraint $\sum_{k=1}^{N_{e}} w_{k}=1$. Note that all involved distributions need only to be available up to normalisation constants which do not depend on $m$ or $\delta$.

The subsequent discussion will reveal a natural choice for $\pi_{\Delta}(\delta \mid m)$ and will lead to an explicit expression for Eq. 11 . Let us therefore go through the analysis to factor

$p_{M \Delta}(m, \delta)=n\left(m^{\prime}\right)^{-1} q_{M^{\prime} \Delta^{\prime}}\left(m^{\prime}, \delta^{\prime}\right) J(m, \delta)$

to determine a candidate $\pi_{\Delta}(\delta \mid m)$. First, we expand the negative $\log$ density $-\log q_{M^{\prime} \Delta^{\prime}}$, ignoring the normalization constant:

$$
\begin{aligned}
& \frac{1}{2}\left(m-\bar{m}+C_{M} G^{T} C_{D}^{-1}(g(m)-\delta)\right)^{T} C_{M}^{-1}\left(m-\bar{m}+C_{M} G^{T} C_{D}^{-1}(g(m)-\delta)\right) \\
& +\frac{1}{2}\left(\delta-d^{o}\right)^{T} C_{D}^{-1}\left(\delta-d^{o}\right) \\
& =\frac{1}{2}(m-\bar{m})^{T} C_{M}^{-1}(m-\bar{m})+\frac{1}{2}(g(m)-\delta)^{T} C_{d}^{-1} G C_{M} G^{T} C_{D}^{-1}(g(m)-\delta) \\
& +\frac{1}{2}(g(m)-\delta)^{T} C_{d}^{-1} G(m-\bar{m})+\frac{1}{2}(m-\bar{m})^{T} G^{T} C_{D}^{-1}(g(m)-\delta) \\
& +\frac{1}{2}\left(g(m)-d^{o}\right)^{T} C_{D}^{-1}\left(g(m)-d^{o}\right)+\frac{1}{2}(g(m)-\delta)^{T} C_{D}^{-1}(g(m)-\delta) \\
& -\frac{1}{2}(g(m)-\delta)^{T} C_{D}^{-1}\left(g(m)-d^{o}\right)-\frac{1}{2}\left(g(m)-d^{o}\right)^{T} C_{D}^{-1}(g(m)-\delta) .
\end{aligned}
$$

To simplify the notation we will use

$$
V:=C_{D}+G C_{M} G^{T}
$$

and

$$
\eta(m):=G(m-\bar{m})-\left(g(m)-d^{o}\right) .
$$

Then, using the new definitions to simplify notation, we obtain

$$
\begin{gathered}
\overbrace{A_{0} \exp \left[-\frac{1}{2}(m-\bar{m})^{T} C_{M}^{-1}(m-\bar{m})-\frac{1}{2}\left(g(m)-d^{o}\right)^{T} C_{D}^{-1}\left(g(m)-d^{o}\right)\right]}^{p_{M}(m, \delta)=} \\
\overbrace{A_{A_{1}|V|^{1 / 2} \exp \left[-\frac{1}{2}\left(\delta-g(m)-V^{-1} \eta(m)\right)^{T} V\left(\delta-g(m)-V^{-1} \eta(m)\right)\right]}^{\pi_{\Delta}(\delta)}}^{\pi^{(m)}} \\
\times n\left(m^{\prime}\right)^{-1} A_{2}|V|^{-1 / 2} \exp \left[\frac{1}{2} \eta(m)^{T} V^{-1} \eta(m)\right] J(m, \delta),
\end{gathered}
$$

where $A_{0}, A_{1}$, and $A_{2}$ are all normalisation constants, independent of $m$ and $\delta . A_{0}$ is determined from the requirement that $\int \pi_{M}(m) \mathrm{d} m=1$. Similarly, $A_{1}$ is determined from the requirement that $\int \pi_{\Delta}(\delta \mid m) \mathrm{d} \delta=$ 1. Finally, $A_{2}$ is determined from the requirement that $\int p_{M \Delta}(m, \delta) \mathrm{d} m \mathrm{~d} \delta=1$. The last line of Eq. 14 is exactly the difference between the proposal density and the target density, which determines the importance weights Eq. 11.

Note that if the observation operator is linear, then $n\left(m^{\prime}\right)=1$ and all terms on the last line of Eq. 14 are independent of $m$ so the target and proposal densities are equal: $p_{M \Delta}=q_{M \Delta}$. 


\subsection{Weighting of RML samples}

To weight the RML samples, we compute the weights by $w_{k} \propto \frac{\pi_{M}\left(m_{k}\right) \pi_{\Delta}\left(\delta_{i} \mid m_{k}\right)}{p_{M \Delta}\left(m_{k}, \delta_{k}\right)}$

with $\pi_{\Delta}(\delta \mid m)$ as defined in Eq. 14. So the weight on a sample is

$w \propto n\left(m^{\prime}\right)|V|^{1 / 2} \exp \left[-\frac{1}{2} \eta(m)^{T} V^{-1} \eta(m)\right] J^{-1}(m, \delta)$.

The Jacobian determinant and the gradient of the misfit term with respect to the parameter are necessary for the computation of weights. For the low-dimensional space, it is easy to calculate them. However, the computation of the Jacobian determinant and the gradient is difficult when the problems are strongly nonlinear.

In this section, we use the low-rank approximation to get the Jacobian determinant and $\operatorname{det} V$. Using the GaussNewton approximation for the Jacobian matrix given by Eq. 10, we have

$J(m, \delta) \approx\left|I+C_{M} G^{T} C_{D}^{-1} G\right|$

and $J$ becomes independent of $\delta$. Let $m_{\mathrm{MAP}}$ and $H_{\text {misfit }}$ denote the minimizer point of Eq. 6 and the Hessian matrix of $L_{i}(m)$ with respect to the misfit term at $m_{\mathrm{MAP}}$, respectively. Thus the Hessian matrix of $L_{i}(m)$ at $m_{\mathrm{MAP}}$ is given by

$H_{\text {map }}=C_{M}^{-1}+H_{\text {misfit }}$.

To compute its determinant, we would like to approximate $H_{\text {map }}$ with a relatively small number of terms. Thus we solve the following generalized eigenvalue problem (GEP): find $U \in \mathbb{R}^{N_{m} \times N_{m}}$ and $\Lambda=\operatorname{diag}\left(\lambda_{i}\right) \in \mathbb{R}^{N_{m} \times N_{m}}$, which are the generalized eigenvectors and eigenvalues of the matrix pair $H_{\text {misfit }}$ and $C_{M}^{-1}$, respectively:

$H_{\text {misfit }} U=C_{M}^{-1} U \Lambda$,

such that

$U^{T} C_{M}^{-1} U=I \quad$ and $\quad H_{\text {misfit }}=C_{M}^{-1} U \Lambda U^{T} C_{M}^{-1}$.

For the large-scale flow problem, we consider the Whittle-Matérn prior covariance operator based on the inverse of an elliptic differential operator,

$$
\begin{aligned}
\mathcal{C}_{\text {prior }} & =(-\gamma \Delta+\alpha I)^{-2}, \\
& =\frac{1}{4 \pi \gamma \alpha}\left(\frac{r}{\sqrt{\gamma / \alpha}}\right) K_{1}\left(\frac{r}{\sqrt{\gamma / \alpha}}\right)
\end{aligned}
$$

where $K_{1}$ denotes the modified Bessel function of the second kind of order 1 . Equation 16 provides a sparse representation of the inverse covariance and a square root factorization that is useful for computing a low-rank approximation of the Hessian matrix [8]. From Eq. 16 the variance is seen to be $(4 \pi \alpha \gamma)^{-1}$ and the range of the covariance to be proportional to $\sqrt{\gamma / \alpha} . C_{M}$ is given by the discretization of $\mathcal{C}_{\text {prior }}$ and the inverse of $C_{M}$ can be easily factored

$C_{M}^{-1}=Q Q^{T}$.

So we have

$H_{\text {map }}=Q Q^{T} U \Lambda U^{T} Q Q^{T}+Q Q^{T}=Q \hat{U}(\Lambda+I) \hat{U}^{T} Q^{T}$,

where $\hat{U}=Q^{T} U$ is the matrix of orthonormal eigenvectors for $Q^{-1} H_{\text {misfit }} Q^{-\mathrm{T}}$. We actually want the determinant

$\left|C_{M} H_{\text {map }}\right|=\left|Q^{-1} H_{\text {map }} Q^{-T}\right|=\left|\hat{U}(\Lambda+I) \hat{U}^{T}\right|=\prod_{i=1}^{N_{m}}\left(1+\lambda_{i}\right)$.

When the generalized eigenvalues $\left\{\lambda_{i}\right\}$ decay rapidly, we can use a low-rank approximation of $H_{\text {misfit }}$ by retaining only the $r$ largest eigenvalues and corresponding eigenvectors, i.e.,

$H_{\text {misfit }} \approx Q Q^{T} U_{r} \Lambda_{r} U_{r}^{T} Q Q^{T}$.

Thus we have

$J(m, \delta) \approx \prod_{i=1}^{r}\left(1+\lambda_{i}\right)$

We also need the determinant of $V$ for the computation of weights in Eq. 15. In Section 3.3, we will use a diagonal matrix for $C_{D}$, i.e., $C_{D}=\sigma^{2} I$. Due to $H_{\text {misfit }} \approx G^{T} C_{D}^{-1} G$, we have

$G^{T} \sigma^{-2} I G \approx C_{M}^{-1} U \Lambda U^{T} C_{M}^{-1}$.

Then

$C_{M} G^{T} G \approx \sigma^{2} U \Lambda U^{T} C_{M}^{-1}$.

The determinant of $V$

$$
\begin{aligned}
& |V|=\left|\sigma^{2} I+G C_{M} G^{T}\right| \\
& =\sigma^{2\left(N_{d}-N_{m}\right)}\left|\sigma^{2} I+C_{M} G^{T} G\right| \\
& \approx \sigma^{2\left(N_{d}-N_{m}\right)}\left|\sigma^{2} I+\sigma^{2} U \Lambda U^{T} C_{M}^{-1}\right| \\
& =\sigma^{2 N_{d}}\left|I+Q^{T} U \Lambda U^{T} Q\right| \\
& =\sigma^{2 N_{d}}\left|I+\hat{U} \Lambda \hat{U}^{T}\right| \approx \sigma^{2 N_{d}} \prod_{i=1}^{r}\left(1+\lambda_{i}\right) .
\end{aligned}
$$

Thus the determinant of $V$ can be also replaced by a lowrank approximation. $\sigma^{2 N_{d}}$ is not necessary in $|V|$ because it appears in all weights and can be factored out. Then Eq. 15 can be approximated by

$w \propto n\left(m^{\prime}\right) \exp \left[-\frac{1}{2} \eta(m)^{T} V^{-1} \eta(m)\right] \prod_{i=1}^{r}\left(1+\lambda_{i}\right)^{-1 / 2}$.

In the approach described above, the computation of $G$ for the weights is necessary. To obtain $G$ for the flow problem in Section 3.3, we solve an adjoint system [42]. In Section 3.1, we investigate the effect of a Gauss-Newton approximation for the weights for cases in which all the critical points and only minimizers of Eq. 6 are obtained, 
respectively. As the Gauss-Newton approximation of the weights is shown to be poor for maximizers of the objective function, we only consider the minimizers in the Darcy flow example (Section 3.3).

\subsection{Weighted RML sampling algorithm}

In this section, we consider two possible situations when seeking independent samples from Eq. 2 for a log-posterior of the form Eq. 3. In both cases, we allow for the possibility that the stochastic cost function Eq. 5 is nonconvex. Note that in this case the number of critical points may be greater than 1. In the first algorithm, we assume that all critical points can be identified, while in the second case, we suppose that it is only possible to identify a single critical point, but that the total number of critical points is unknown.

\subsubsection{All critical points found}

For problems in low dimensions, with polynomial observation operators $g$, or convex cost functions, it may be feasible to find all critical points for each pair $\left(m^{\prime}, \delta^{\prime}\right)$. If the $i$ th sample of $\left(m^{\prime}, \delta^{\prime}\right)$, generates a cost function $L_{i}(m)$ with $n_{c_{i}}$ critical points, it is possible to sample correctly by weighting each critical point using Eq. 15.

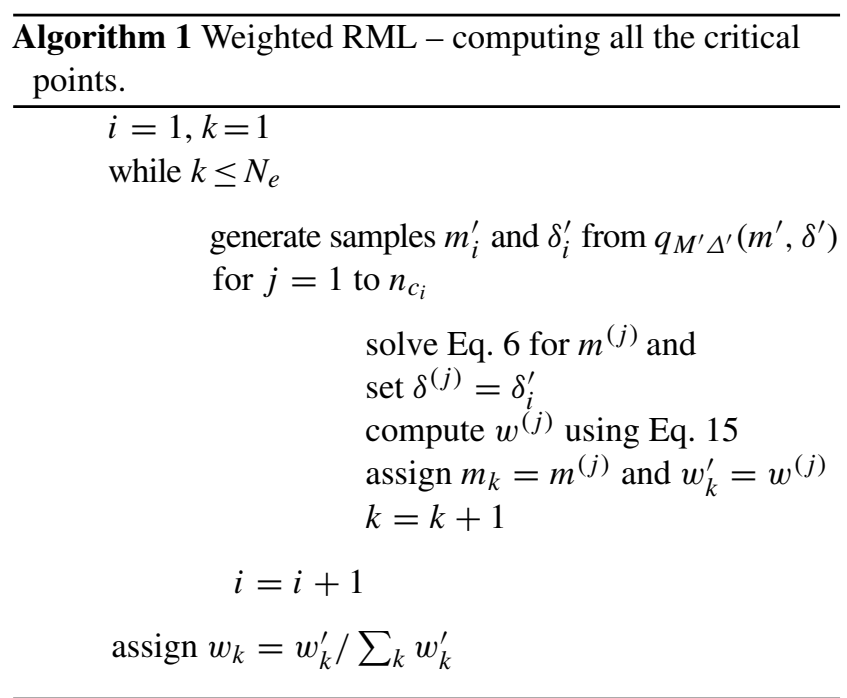

Note that when the forward operator $g$ is linear, i.e., $g(m)=G m$, the stochastic cost function is convex for each pair $\left(m^{\prime}, \delta^{\prime}\right)$. The log-posterior has a single critical point, which is the minimizer. Thus, for linear forward operators, we just need to compute the minimizers and the weights are all equal by using Eq. 15 .

\subsubsection{One critical point found}

Finding all the critical points is not feasible when the problem dimension and the complexity increases. In these cases, it is unlikely that even the number of critical points will be known. Instead of seeking to compute all critical points, we compute a single critical point using a random starting point for the optimization. We assume, without evidence, that the optimization performed this way uniformly samples the critical points, in which case the resulting $\sum_{j=1}^{n_{c}} m^{(j)} w^{(j)}$ provides an unbiased estimator in the same manner as it is obtained from computing all critical points.

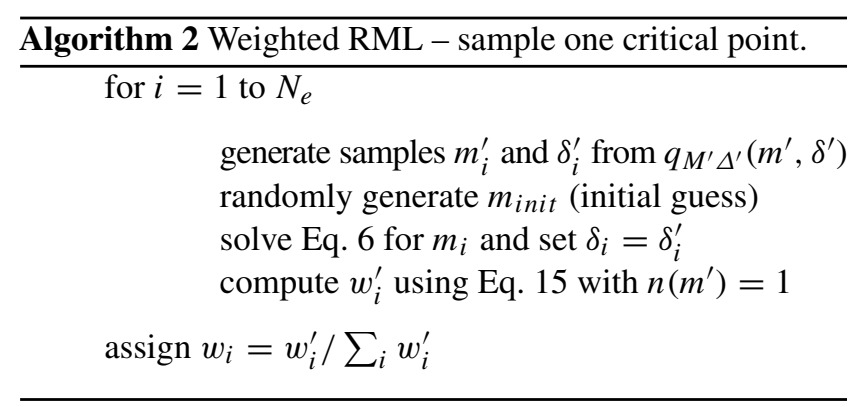

\subsubsection{Local minimizers only}

Due to the high dimension of most realistic applications, solutions of Eq. 6 are much more difficult to obtain than local minimizers of $L$, although local maximizers can clearly be easily found as minimizers of $-L_{i}$. In general, however, it appears that in high dimensions, minimizers of $L_{i}$ are far more important than maximizers. When that is the case, a Gauss-Newton approximation of the Jacobian determinant can be made with little loss of accuracy

$$
\begin{aligned}
J & =\left|I+D\left(C_{M} G^{T} C_{D}^{-1}(g(m)-\delta)\right)\right| \\
& \approx\left|I+C_{M} G^{T} C_{D}^{-1} G\right| .
\end{aligned}
$$

Thus, in the Darcy flow problem, we will solve for random minimizers instead of solving for random critical points and we will compute a Gauss-Newton approximation of the Jacobian determinant. The consequence of this approximation is that the distribution of samples will not be exact. In Section 3.1, we investigate numerically the consequence of sampling only the minimizers.

To obtain the weights, the computation of the Jacobian determinant and the gradient of the misfits with respect to the parameter are necessary. For the first two examples, it is easy to compute the weights. For the large-scale flow problem, we use the Gaussian-Newton method in the hIPPYlib [42] to get a low-rank approximation of the Jacobian determinant. This can reduce the cost.

\section{Numerical examples}

In this section, we present sampling results using test cases of increasing size and complexity. We first demonstrate the 
methodology using a toy problem that has been previously used by [44]. It is small enough that the true posterior distribution is easily derived. We focus on the parameter values that are difficult to sample correctly. This example shows the difficulty with using only the minimizers and not accounting for the limited range in the solutions.

A second simple example is the "banana-shaped" distribution from [18]. It has been used fairly often to test adaptive forms of MCMC [12, 19, 30, 37, 43]. It is simple enough that computing the Jacobian determinant is not a challenge so the focus again is on showing that if we find the roots and compute the weights, the sampling is correct.

\subsection{Bimodal posterior pdf}

The first example has been previously discussed in [44] where it was used to demonstrate properties of the randomized maximum a posteriori sampling algorithm. One of their test problems required sampling from the distribution

$\pi_{M}(m) \propto \exp \left(-\frac{1}{2}(m-0.8)^{2}-\frac{1}{2 \sigma_{d}^{2}}\left(m^{2}-1\right)^{2}\right)$.

Although [44] used three different values of $\sigma_{d}$, we only show results for the most difficult value, $\sigma_{d}=0.5$. For larger and smaller values, the posteriori distribution is more easily modeled as a mixture of Gaussians and is therefore easier to sample.

In our approach, approximate samples from the posteriori distribution are obtained by solving for the critical points of

$L_{i}(m)=\frac{1}{2}\left(m-m_{i}^{\prime}\right)^{2}+\frac{1}{2 \sigma_{d}^{2}}\left(m^{2}-\delta_{i}^{\prime}\right)^{2}$

$m_{i}^{\prime} \sim N(0.8,1) \quad \delta_{i}^{\prime} \sim N(1,0.25)$.

Because the objective function in this case is a polynomial, it is straightforward to obtain all real roots of Eq. 6. For most choices of $\left(m^{\prime}, \delta^{\prime}\right)$ there are three real roots $-N_{s}=$ 10,000 samples of $\left(m^{\prime}, \delta^{\prime}\right)$ from the prior generated $N_{e}=$ 25,046 pairs of $(m, \delta)$. The locations of the roots are shown in Fig. 1a. The set of points in the center of the plot correspond to maximizers of Eq. 17. The points on the right side correspond to the global minimum, and the points on the left correspond to the local minimum. The colors show unnormalized importance weights for each sample. The maximizers are generally given small weights (Fig. 1b), although a small number of maximizers have weights that are similar to the weights of points near the local minimum.

Because the samples are generated independently (or in groups of three), the quality of the weighted sampling approximation to the target distribution is limited only by sampling error - larger samples provide better approximations. Figure 2 shows results for three different sample sizes $N_{s}(200,1000,5000)$. The number of weighted samples is larger than the ensemble size because a single sample from the prior usually results in three critical points.

For this problem, computing all real roots of (6) and computing importance weights for each root is trivial. For large high-dimensional problems, finding multiple roots and computing the weights will be challenging. Here we examine the consequence of three realistic approximations to correct sampling: (1) identifying only the minimizers of the cost function, (2) using a Gauss-Newton (GN) approximation of the Jacobian of the transformation and (3) neglecting importance weights altogether. Figure $3 \mathrm{c}$ shows that correct sampling of the target distribution is obtained when all critical points are included and the weights are computed accurately. If the importance samples are neglected (Fig. 3a) or if the GN approximation of the Jacobian is used (Fig. 3b), the distribution of samples is badly distorted. When it is not possible to compute the Jacobian accurately in high dimensions, it appears to be advisable to only compute the minimizers. The distribution of samples obtained using the GN approximation applied to minimizers (Fig. 3e) is nearly as good as the results with correct weights, and far better than results with no importance weighting.

\subsection{Banana-shaped posterior pdf}

The second numerical example is the widely used "bananashaped" target density initially presented in [18], but extended to higher dimensions by [37],

$$
\begin{aligned}
\pi_{M}(m) \propto \exp [ & \left.-\frac{1}{2 \sigma_{m}^{2}}\left(m_{1}^{2}+m_{2}^{2}+\cdots+m_{N_{m}}^{2}\right)\right] \\
& \times \exp \left[-\frac{1}{2 \sigma_{d}^{2}}\left(4-10 m_{1}-m_{2}^{2}\right)^{2}\right]
\end{aligned}
$$

with $\sigma_{d}=4$ and $\sigma_{m}=5$. In our numerical experiment, we use parameters values from [30], but increased the dimension of $m$ to 4 . The first term of Eq. 18 is identified as the Gaussian prior with model covariance $C_{M}=I$ and the second term as the log-likelihood, with

$g(m)=10 m_{1}+m_{2}^{2}$.

Because of the curved shape of the objective function (Fig. 5b), accurate computation of minimizers of $L_{i}$ was relatively difficult. Three projections of the first 3000 approximate samples obtained using the Broyden-FletcherGoldfarb-Shanno algorithm for minimization are shown in Fig. 4. Each minimization was initiated at the point $m_{i}^{\prime}$.

For this problem, which has a single critical point, the empirical distribution obtained from minimization appears to be relatively good. The true conditional distribution distribution $\pi\left(m_{1}, m_{2} \mid m_{3}=0, m_{4}=0\right)$ is compared in Fig. 5b, with a kernel estimate of the empirical marginal 


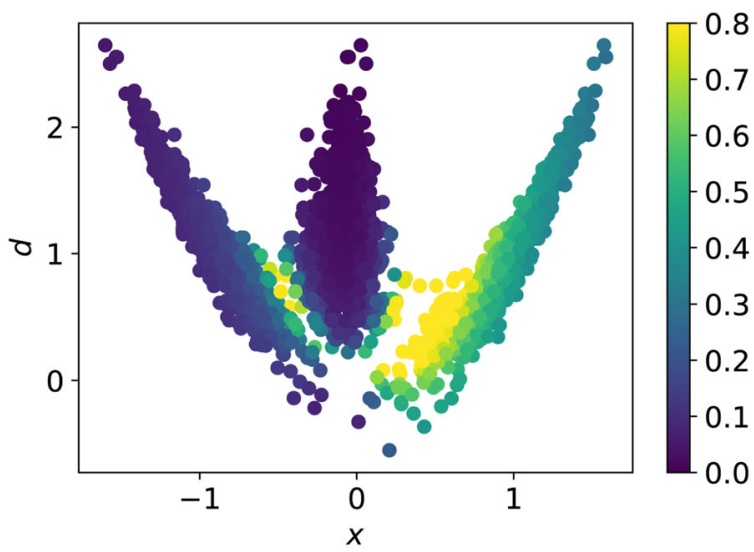

(a) Solutions of $\nabla L_{i}(m)=0$. Color indicates importance weight.

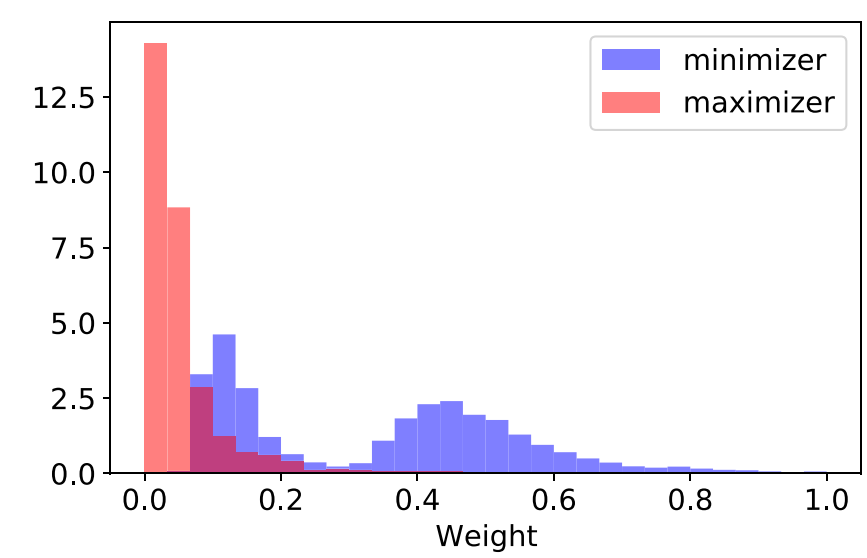

(b) Distribution of weights from critical points.

Fig. 1 Critical points and importance weights for the quadratic observation operator

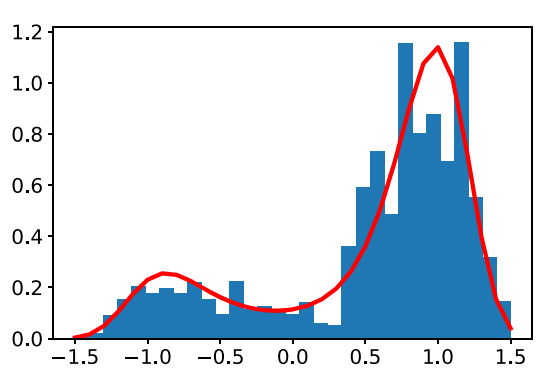

(a) $N_{s}=200$.

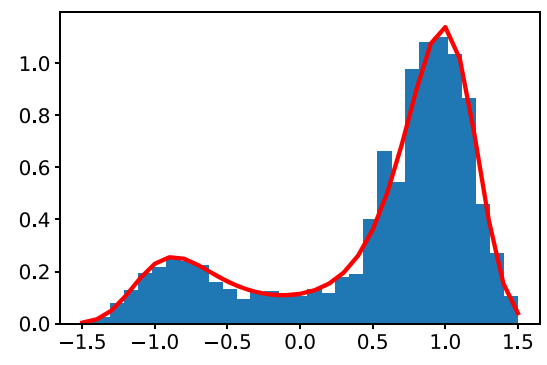

(b) $N_{s}=1000$.

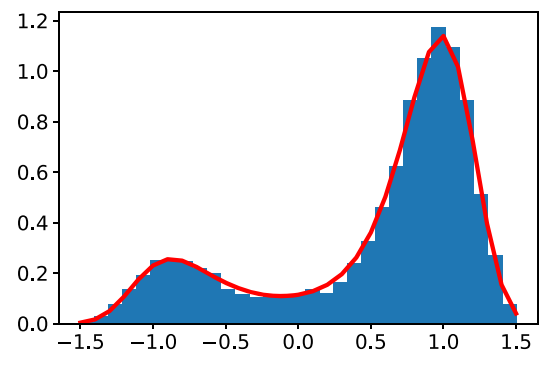

(c) $N_{s}=5000$.

Fig. 2 Weighted sampling approximations to the true posterior distribution
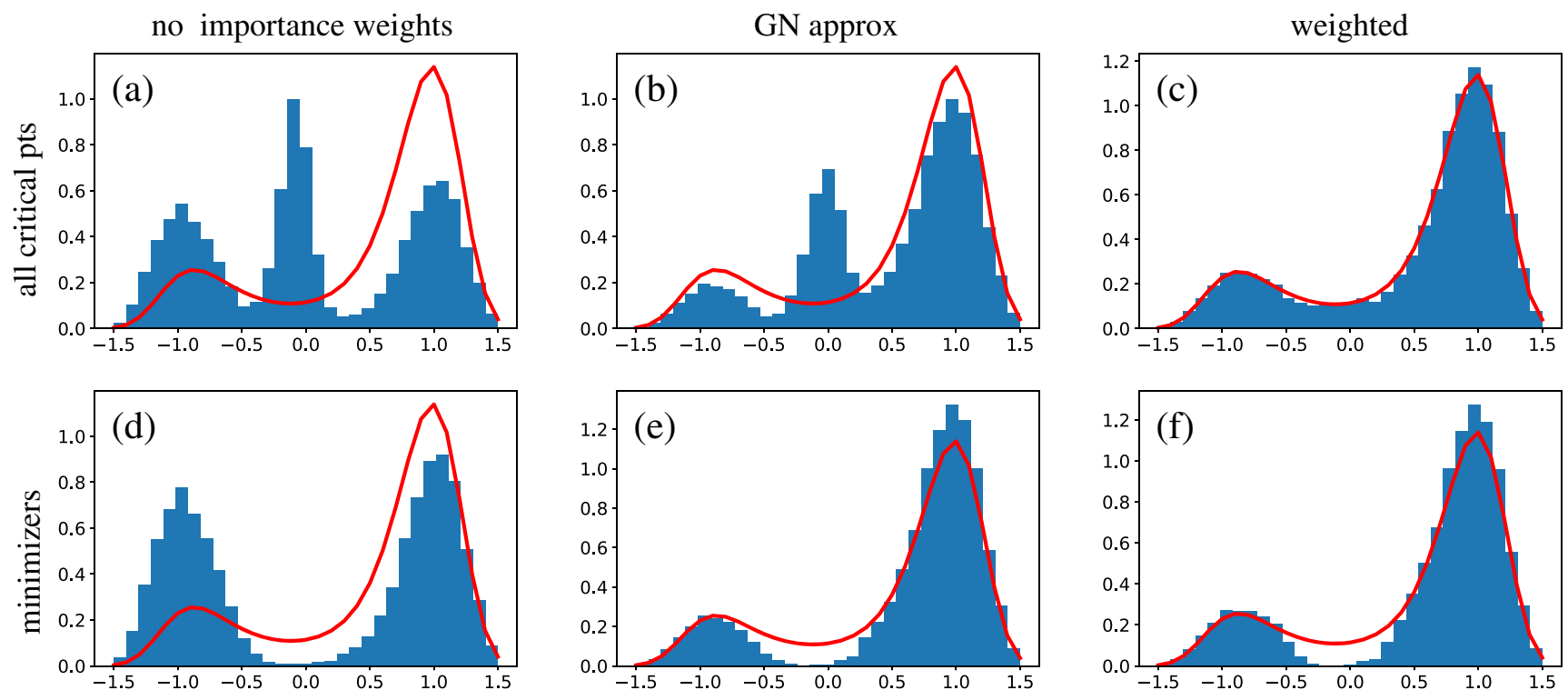

Fig. 3 Compare distributions from approximations to sampling based on computation of critical points of cost function 


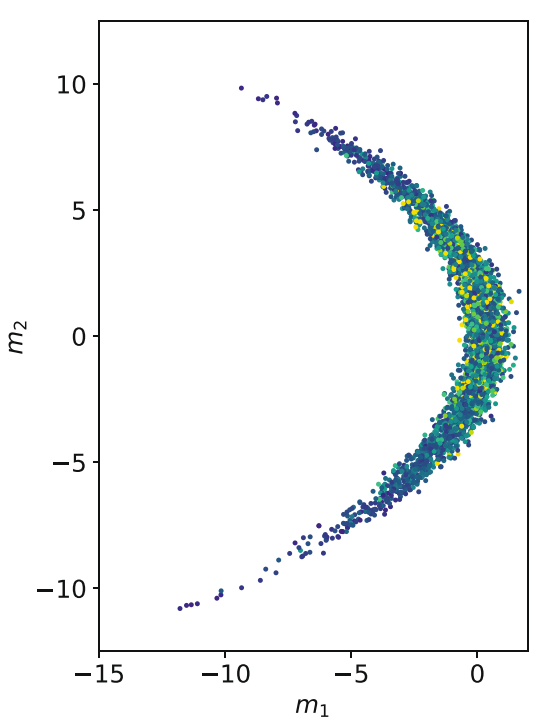

(a) $m_{1}-m_{2}$ plane.

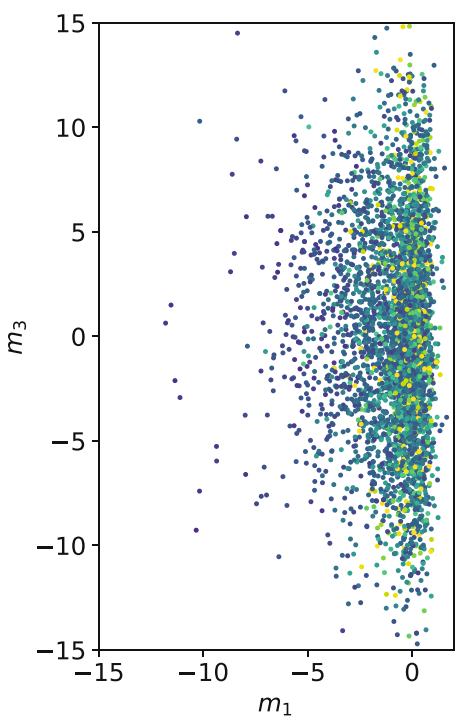

(b) $m_{1}-m_{3}$ plane.

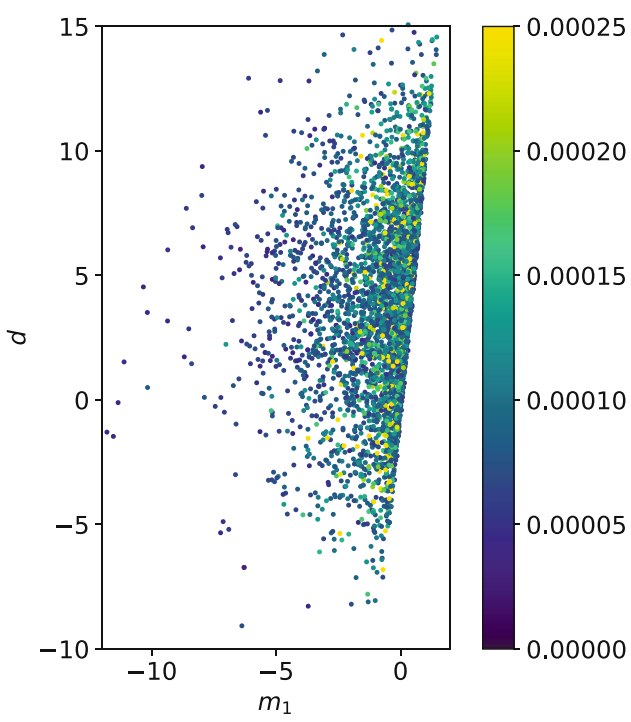

(c) $m_{1}-\delta$ plane.

Fig. 4 Critical points of the banana-shaped objective function. Color indicates importance weight on the samples. The same color scale is applied to all subplots

distribution for $m_{1}, m_{2}$ (dashed contours) obtained using 50,000 minimizations. Also, based on the distribution of unnormalized importance weights (Fig. 5a), it appears that the weights are not dominated by a few large values, which is confirmed by a high effective sampling efficiency, $N_{\text {eff }} / N_{e}=44796 / 50000 \approx 0.9$, based on Kong's estimator Eq. 19,

$N_{\mathrm{Eff}}=\frac{1}{\sum_{k=1}^{N_{e}} w_{k}^{2}}$,

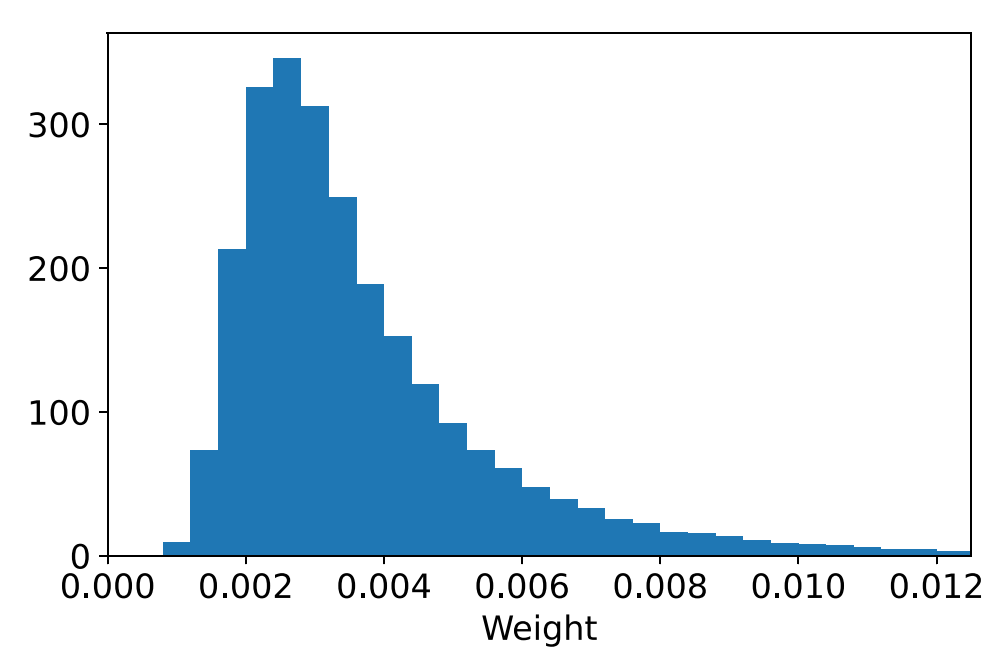

(a) Distribution of importance weights for the minimizers of the log posterior.

$$
\text { the log posterior. }
$$

where $\sum_{k=1}^{N_{e}} w_{k}=1$

\subsection{Darcy flow example}

For the first two examples, there would be no advantage in using RML for sampling - MCMC with a carefully chosen transition kernel would probably be a better alternative in either case. The advantage for RML occurs in large highdimensional problems for which traditional methods are impractical. In this section, we investigate the ability to

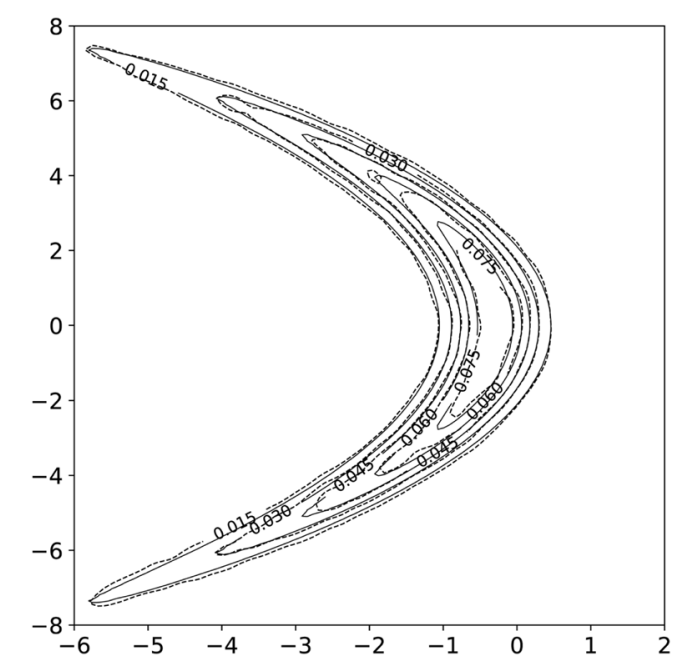

(b) Compare true distribution with estimation from weighted minimizers $\left(m_{1}\right.$ $m_{2}$ plane).

Fig. 5 Minimizers of the banana-shaped objective function 
quantify uncertainty in the permeability field $\kappa(x)$ from spatially distributed observation of steady-state pressure. The pressure $u(x)$ in this example is governed by the equation

$-\nabla \cdot(\kappa(x) \nabla u(x))=0 \quad$ in $\quad \Omega=[0,1] \times[0,1]$

with the mixed boundary conditions

$$
\left\{\begin{aligned}
\nabla u \cdot \mathbf{n} & =0 \quad \text { on } \quad \Gamma_{N_{1}}=0 \times[0,1] \cup 1 \times[0,1] \\
\nabla u \cdot \mathbf{n} & =v(x) \quad \text { on } \quad \Gamma_{N_{2}}=[0,1] \times 1 \\
u(x) & =0 \quad \text { on } \quad \Gamma_{D}=[0,1] \times 0 .
\end{aligned}\right.
$$

As permeability is a positive quantity, it cannot be modelled as a Gaussian random variable. Here, we evaluate sampling with three possible prior distributions for permeability. In all cases, we define a latent variable $m(x)$ that is multivariate Gaussian, with a prior given by Eq. 16 . We take $\alpha=$
0.12 and $\gamma=1.12$ which results in a correlation length of approximately 2 and a variance of 1 . In the first case (Case 1), permeability is modeled as being log-normally distributed, i.e., $\kappa(x)=\exp (m(x))$, which is a typical assumption for the distribution of permeability within a single rock type [15]. In more complex formations, it is often useful to model permeability as being largely determined by rock type. In that case, permeability might be largely uniform within a rock type, but variable between rock types. We created two soft thresholding transformations to model the distribution of permeability in a formation with three rock types. In the the first of the distributions (Case 2), the permeability is related to the latent variable through a highly nonlinear, but monotonic transformation

$\kappa(x)=\exp (\tanh (4 m(x)+2)+\tanh (4 m(x)-2))$.

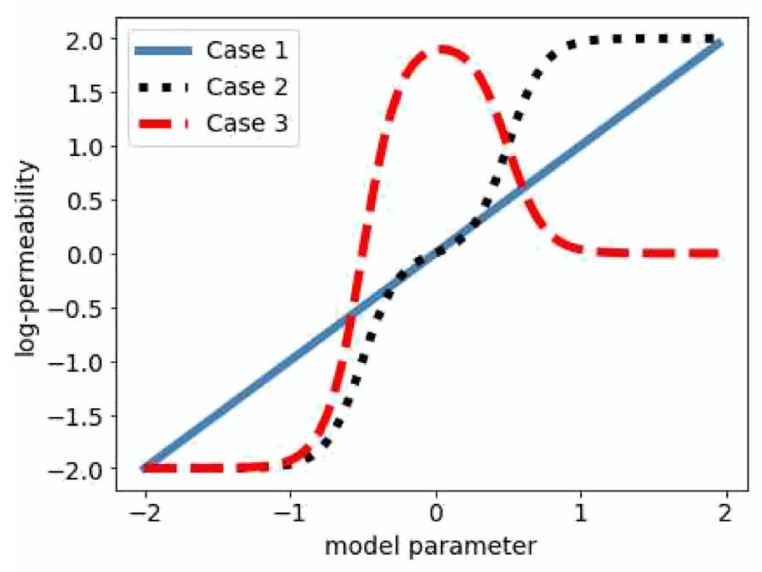

(a) Three transformations

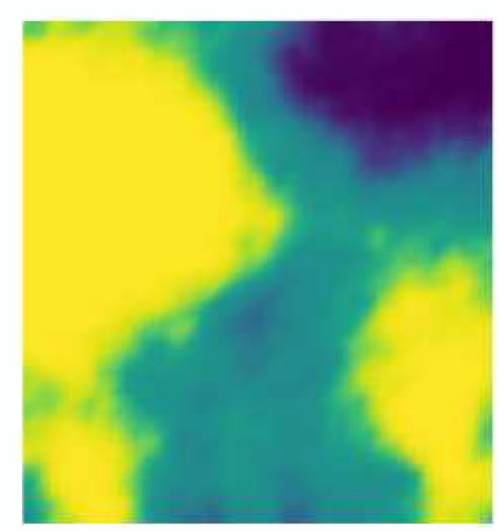

(c) Case 2
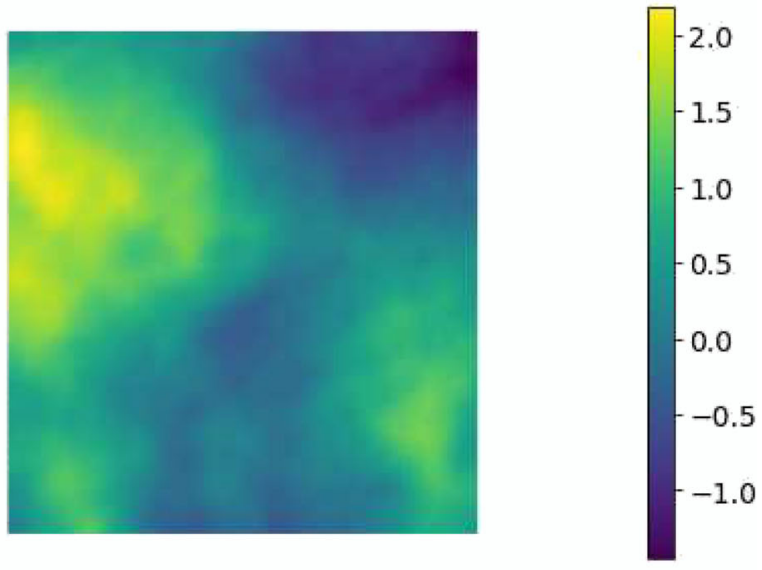

(b) Case 1
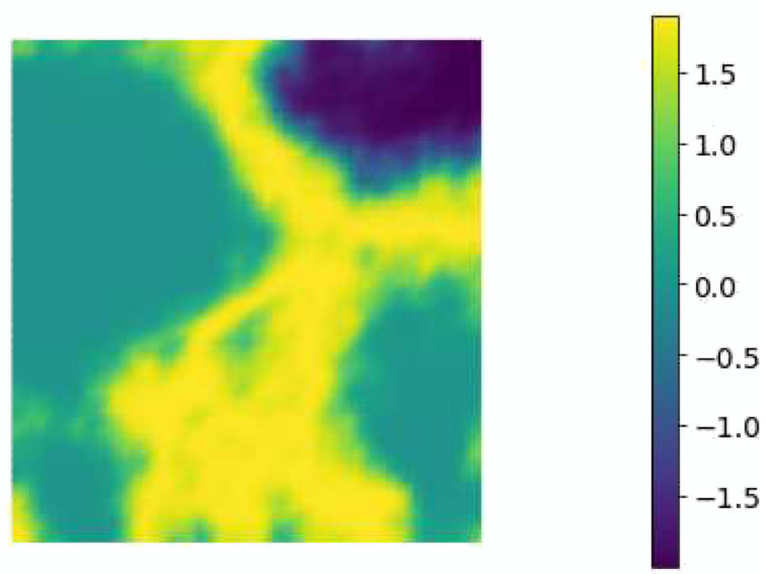

(d) Case 3

Fig. 6 The true log-permeability fields for Cases 1,2 and 3. All cases use the same true latent variable field 


\section{True State}

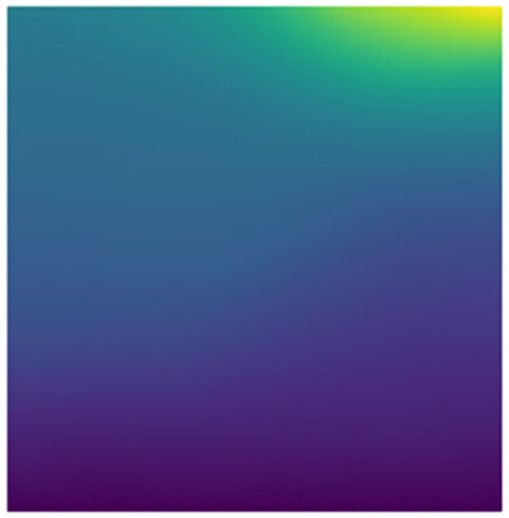

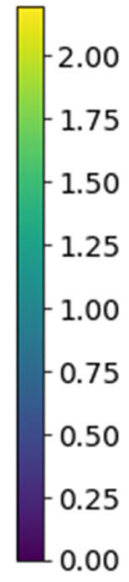
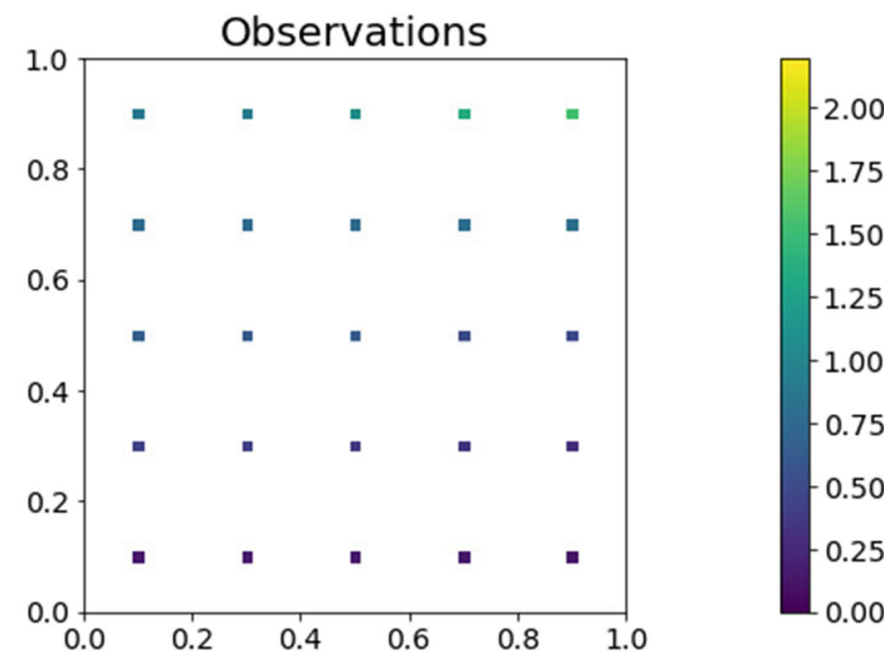

Fig. 7 The true state (left) and observation locations (right)

In the second distribution (Case 2), the permeability is related to the latent variable through a non-monotonic transformation

$\kappa(x)=\exp (2 \tanh (4 m(x)+2)+\tanh (2-4 m(x))-1)$,

which gives a permeability field with a low permeability 'background' and connected high perm 'channels' as might occur in subsurface rock formations [2]. Figure 6 shows the transformations and the three synthetic true logpermeability fields that are used to generate observations for Case 1 (top right), Case 2 (lower left) and Case 3 (lower right).

Figure 7 (left) shows the true pressure field for Case 1. The pressure distributions for Cases 2 and 3 look similar. For each case, we take 25 pressures as observations. The observation locations are distributed on the uniform $5 \times 5$

grid of the domain $[0.1,0.9] \times[0.1,0.9]$ as shown in Fig. 7 (right). The noise in the observations is assumed to be Gaussian and independent with standard deviation 0.01. For the mixed boundary condition, we take $v(x)=2$ for Case 1 and 0.7 for Case 2. Here the piecewise quadratic finite element is used for the state and adjoint spaces, while piecewise linear finite element is used for the parameter space. The forward model is solved by the finite element method with a uniform $50 \times 50$ grid for the three cases. Thus the dimension of the discrete state and adjoint space is 10201 and the dimension of the parameter space is 2601.

We used the hIPPYlib environment [41, 42] for computation of low-rank approximations of eigenvalues of the Hessian. hIPPYlib builds on FEniCS [22, 27] for the discretization of the PDE and uses PETSc [1, 47] for scalable and efficient linear algebra operations and solvers. Minimizers of the objective functions are computed using
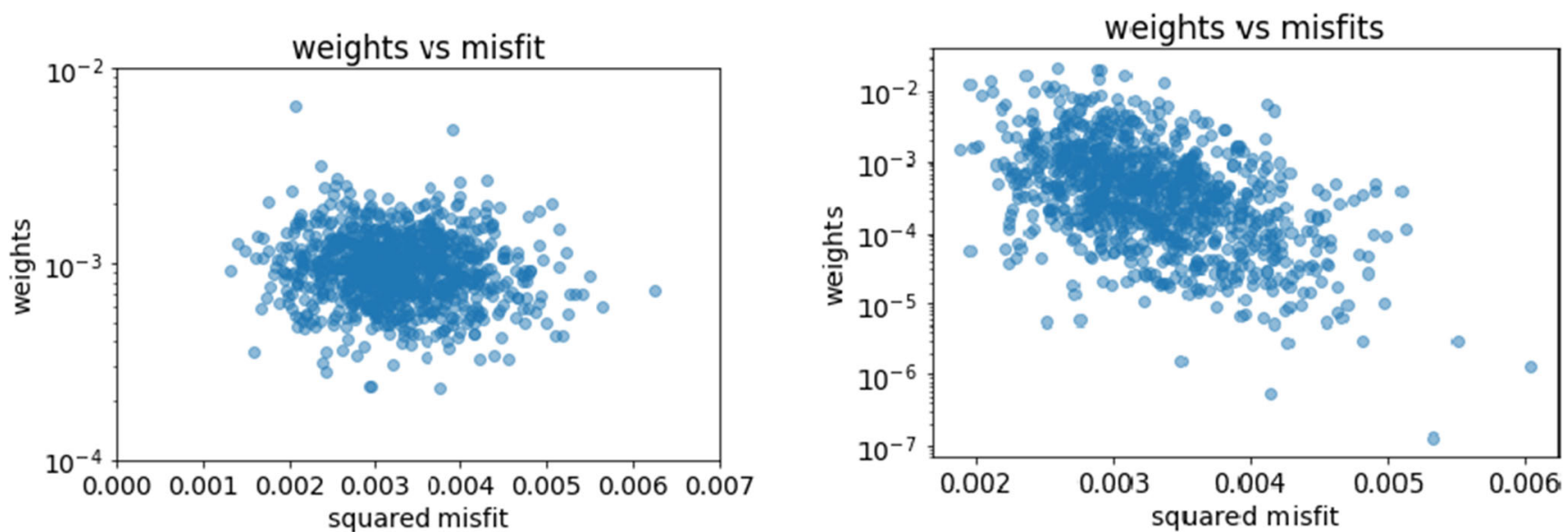

Fig. 8 The weights vs misfits for the minimizers. Blue points show computed weights 
an inexact Newton-CG solver. We used default parameters for minimization, except that we increased the maximum number of iterations to 300 . The actual average number of iterations required for convergence varied considerably for the three cases. In Case 1, an average of 24 iterations were required, Case 2 required 34 iterations and Case 3 required an average of 73 iterations.

For the Darcy flow examples, the low-rank approximation of the Jacobian determinant $J$ was used to reduce the cost of computation of weights. The low-rank approximation adopts the Gauss-Newton method in the hIPPYlib [42]. To illustrate the performance of the proposed method, we compare the results obtained by using the stochastic Newton method [29] implemented in hIPPYlib with that of unweighted RML and weighted RML. Here the stochastic Newton samples are generated from the Gaussian approximation of the posterior. The observation data, prior covariance operator and sample size $N_{e}$ are the same for the RML and stochastic Newton methods. For convenience, we write the stochastic Newton as SN, unweighted RML as RML and weighted RML as WeRML in the figures of the three cases.

\subsubsection{Case 1: permeability field is log-normal}

As the permeability transformation, $\kappa=\exp m$, is monotonic in this example, we might expect the stochastic cost function $L_{i}$ to have a single critical point for each sample from the prior. We confirmed this empirically through an investigation in which we generated a single sample from the prior, but 50 randomly sampled starting points for the minimization. In the experiments, all 50 initial starting models converged to the same model parameters. In the results that we present, RML sampling was performed with 1000 samples from the prior and a single random starting point for each minimization. We dropped 15 out of 1000 samples for which the minimization routine failed to converge to a sufficiently small value of the gradient norm in 300 iterations.

The effective sample size computed from Eq. 19, was relatively high for this case: $N_{\text {eff }} \approx 823$ effective samples. The effective sample efficiency, $N_{\text {eff }} / N_{e}=823 / 985=$ 0.836 . Unlike the toy examples, computing the minimizers and the Jacobian determinant is necessarily approximate in the Darcy flow problem. The expected value for the squared data misfit (with respect to the actual observed values of pressure) is $n_{d} \sigma_{d}^{2}=0.0025$, which is somewhat smaller than the mean of the actual squared misfits, 0.0032 .

Figure 8a shows crossplots of the weights vs squared data misfit computed using $V$ as in Eq. 12. The approximation of log-weights is only slightly correlated with the squared data misfit $(r=-0.09)$ so it appears that in this case, as in the quadratic example, the data mismatch would not serve as a viable surrogate for weighting of samples. In addition to data mismatch, the weights are affected by the nonlinearity of the problem - either through $|V|$ or through the term $(g(m)-G m)$, which occurs in $\eta$.

In Fig. 9, we plot the distribution of sample values of the latent variable at three locations for which the true field has values $m=1.7,0.04$ and -1.17 . For this example, the marginal distributions of samples at observation locations from unweighted RML, SN and weighted RML are all similar and approximately Gaussian.

Estimates of the posterior mean of the log-perm field from three different sampling approaches are shown in Fig. 10. As the permeability field is a monotonic function of the latent variable for this case, the estimated conditional means of the log-perm fields are similar for the three methods.

Despite the similarity of the mean fields and the similarity of the estimates of the posteriori standard deviation from the three methods, the realizations from the three methods are not as similar in their ability to reproduce data (Fig. 11). The mean squared data misfit for weighted RML is 0.0032 , while the mean squared data misfit for stochastic Newton is 0.0048 . This is similar to the observation of Liu and Oliver [25] who showed that the data mismatch of realizations generated from the posteriori mean and covariance in a 1D Darcy flow problem were much larger than data mismatch from MCMC or from RML.

\subsubsection{Case 2: log-permeability is monotonic function of latent variable}

Although it is common to assume that permeability is lognormally distributed within a single rock type, in many subsurface formations the distribution of permeability is largely controlled by 'rock type'. In Case 2 we model the spatial distribution of rock types by applying a soft threshold to a latent Gaussian random field. With this transformation, values of $m<1$ are assigned $\log \kappa \approx-2$ and values values of $m>1$ are assigned $\log \kappa \approx 2$. One practical consequence of this transformation is that minimization of the objective function is more difficult. The second, more important consequence is that the nonlinearity in the neighborhood of the minimizers increases the variability in weights. So while in Case 1, approximately $90 \%$ of the weights were between 0.0006 and 0.0015 , in Case 2 approximately $90 \%$ of the weights fell between $10^{-4}$ and $9 \times 10^{-3}$ (Fig. 8b). The effective sample size for the 930 samples that converged successfully is also smaller in this case; $N_{\text {eff }}=179$ for an efficiency of about $19.2 \%$.

As the posterior distribution is not even approximately Gaussian, the mean and variance may not be the best attributes for judging the quality of the data assimilation. It is common in inverse problems to judge the quality of the realizations by the data misfit after calibration. The expected value of the mean squared data mismatch with 
observations is 0.0025 . The value computed from weighted RML (0.0029) is quite close to that value. In contrast, the value from SN realizations $(0.0087)$ is about 3.5 times larger than expected and the value from unweighted RML (0.0033) is slightly larger than weighted RML. The distributions of squared data misfits for the three methods are shown in Fig. 12a.

\subsubsection{Case 3: log-permeability is non-monotonic function of latent variable}

Applying the transformation Eq. 21, we obtain a permeability field with a low permeability 'background' and connected high perm 'channels'. The 'true' latent variable field and the corresponding true log-permeability field are
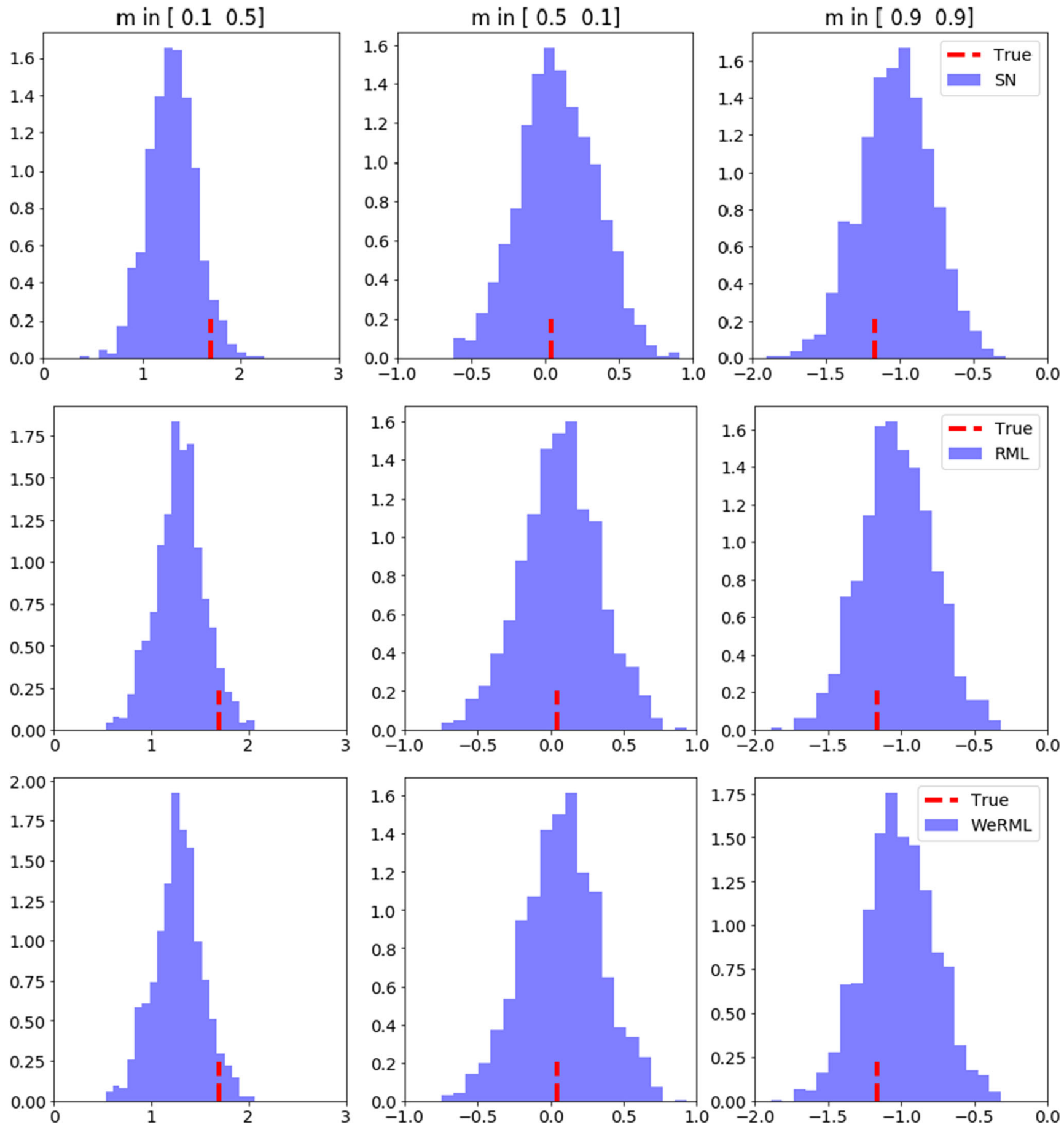

Fig. 9 Density histograms of samples of the latent variable at locations $(0.1,0.5),(0.5,0.1)$ and $(0.9,0.9)$ using SN (upper row), unweighted RML (middle row) and weighted RML (lower row) 
shown in Fig. 6. The true pressure field, from which data are generated, and observation locations are plotted in Fig. 13.

In this example, the transformation from the Gaussian latent variable to the permeability variable is highly nonlinear and non-monotonic, so we should expect to encounter two problems: convergence to the minimizer will be slow [26] and the algorithm is likely to converge to a local minimum that does not have large probability mass associated with it.

We focus on the distribution of samples that are obtained using a practice that could feasibly be applied to largescale subsurface data assimilation problems if a gradient is available - search for a single minimizer for each sample from the prior and use the Gauss-Newton approximation of the Jacobian to compute the weights. Here we have taken 1000 samples from the prior and performed 1000 corresponding minimizations, from which we obtained $N_{e}=885$ samples with successful termination and weights. Using Eq. 19, we compute the effective sample

\section{True parameter}

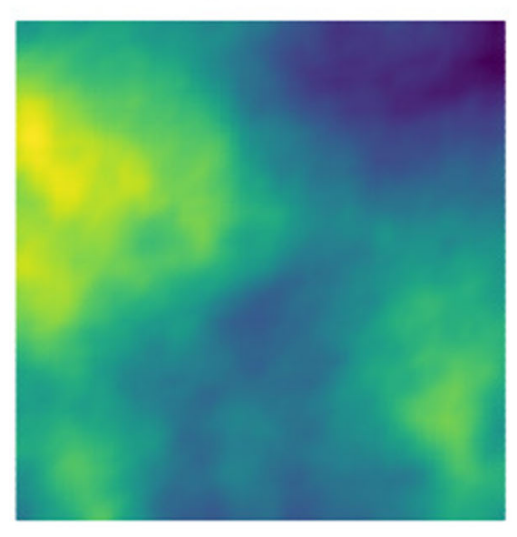

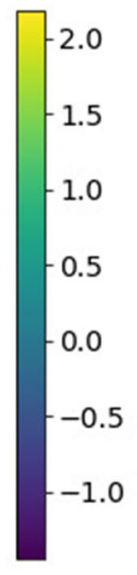

\section{Posterior mean (RML)}

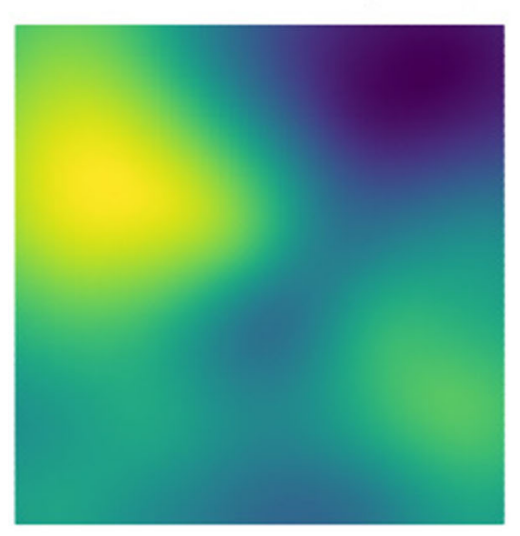

size $N_{\mathrm{Eff}} \approx 14$. Because of the dimensionality of the problem, it is not possible to completely characterize the posterior distribution for either the latent variables, or for the log-permeability. In order to gain some understanding, we examine the marginal distribution of the minimizers at three observation locations for which the true logpermeability values are approximately, $-2,0$ and 2 . The marginal distribution of unweighted minimizers (upper row Fig. 14) is bimodal at two of the observation locations. The lower row of Fig. 14 (lower row) shows the corresponding distribution of unweighted log-permeability values at the same locations. Although the spread of $m$ is fairly large at each of the observation locations, the spread of the log-permeability values are tightly centered on $-2,0$ and 2 in Fig. 14 (lower row). This is a consequence of the thresholding property of the log-permeability transform.

The colors used for the density histograms in Fig. 14 separate the samples into two groups: one in which $w>$ $10^{-9}$ and the second for which $w<10^{-9}$. Note that

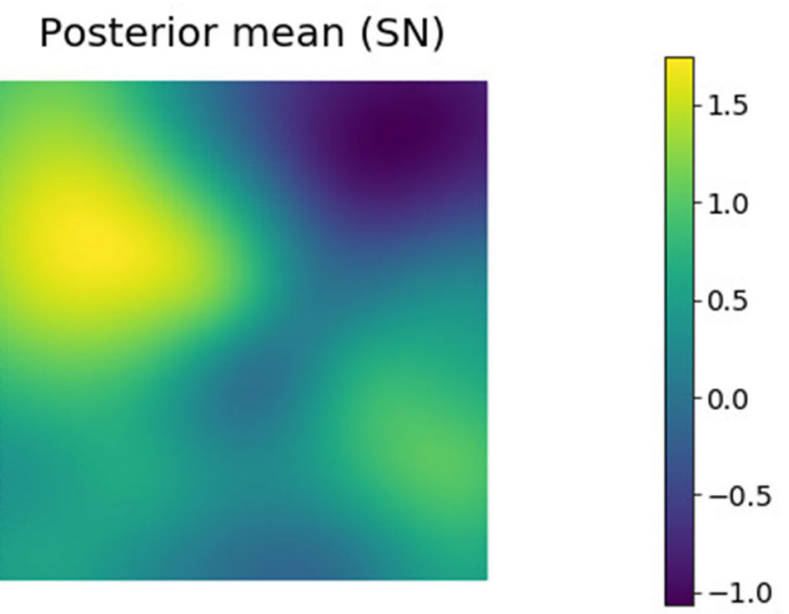

\section{Posterior mean (WeRML)}

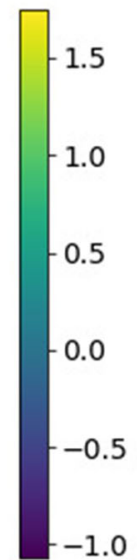

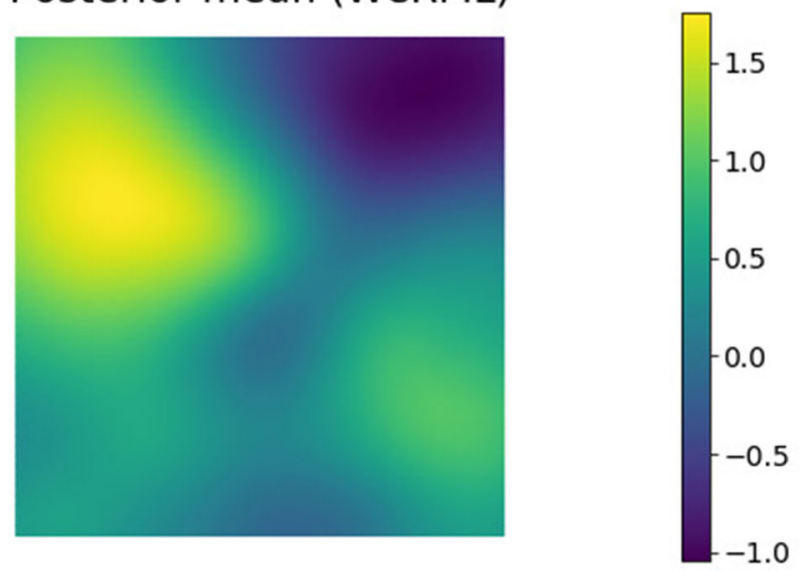

Fig. 10 The true log-permeability field for Case 1 (upper left) and the posterior mean log-permeability fields from three approximate sampling methods: stochastic Newton (upper right), RML without weighting (lower left) and RML with GN approximate weighting (lower right) 


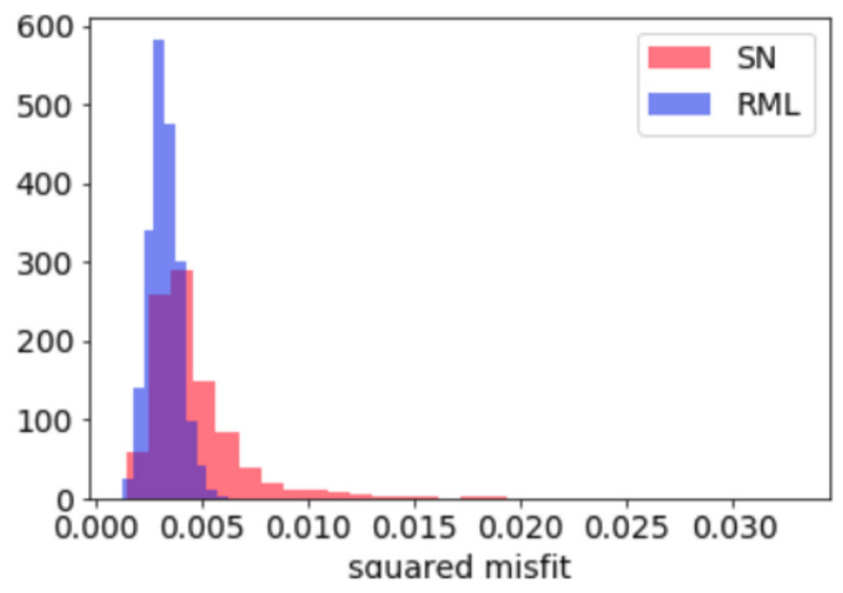

Fig. 11 Distributions of squared data misfit from samples generated using SN and using weighted RML

in Fig. 14 (lower right), a substantial fraction of samples converged to a local minimizer with $\log \kappa \approx 0$ at $x, y=$ $(0.9,0.9)$, which is far from the true value, $\log \kappa^{\text {true }} \approx$ -2 . Many of the clearly erroneous minimizers are easily eliminated, however, by the low weights. Note that the inefficiency of the sampling in this case is a result of the non-monotonic nature of the permeability transform - to get from $\log \kappa=0$, which is a local minimizer, to the correct root $\log \kappa=-2$ it is necessary to pass through the point $\log \kappa=2$. In the histograms, the samples with high weights (blue bars) are generally close to the true values (red dashed line), which is as we expected.

As in the previous case, the expected value of the squared data misfit with the actual observation is approximately 0.0025 at the global minimizer of the stochastic cost function. This is close to the values that are obtained in the best minimizations (Fig. 15 (right)). In this example, however, many of the minimizations converged to minimizers with much larger misfit values (Fig. 15 (left)). The minimizations with large misfit values result from convergence to local minima in the cost function. The number of local minima with large numbers of samples appears to be relatively small as a result of the large correlation range for the latent permeability variable. For this example, it appears that many of the unwanted local minimizers could be eliminated either through the weighting, or through the magnitude of the squared data misfit.

Figure 16 compares distributions of the values of the log-permeability field obtained by the SN and the weighted RML methods at three pressure observation locations. For consistency, the same sample size was used for both methods. The true values of the log-permeability field at the observation locations are close to $-2,0$ and 2 (shown as small red dots). When using the weighted RML samples to approximate the distribution, the samples are seen to be concentrated close to the true values at the three observation locations (Fig. 16 (lower)). Because of the highly nonlinear transformation of the permeability field, the SN method was unable to provide a good approximation to the true posterior distribution, although it did provide plausible estimates of uncertainty in $\log \kappa$ at two of the locations. At the third location (upper left in Fig. 16) the uncertainty in log-permeability was completely misrepresented.

Although the marginal distribution of the logpermeability field is multimodal at some locations before and after data assimilation, the mean of log-permeability is useful for qualitatively gauging the quality of the data assimilation. In Fig. 17, we compare the MAP point of $\mathrm{SN}$, and the unweighted and weighted posterior means of RML with the true log-permeability field. All three methods provide reasonable characterization of the mean log-permeability in the upper right area of the grid, but only

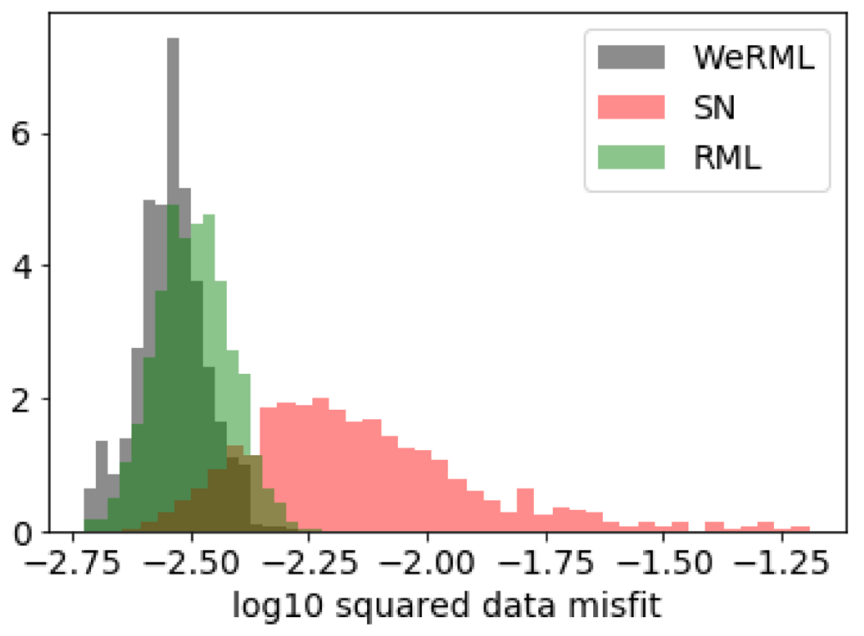

(a) Case 2 (monotonic log-permeability)

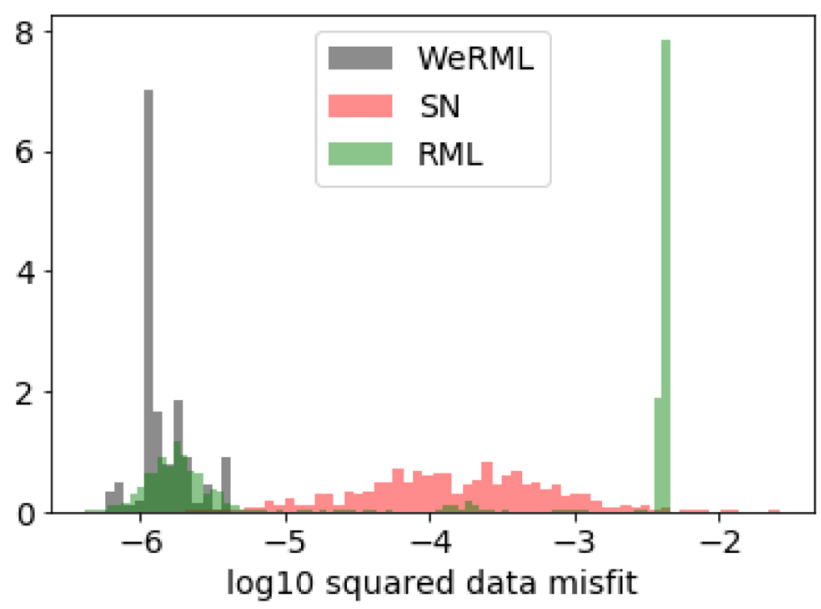

(b) Case 3 (non-monotonic log-permeability)

Fig. 12 Compare distributions of squared data misfit for three sampling methods: RML, weighted RML (WeRML), and stochastic Newton (SN) 


\section{True State}

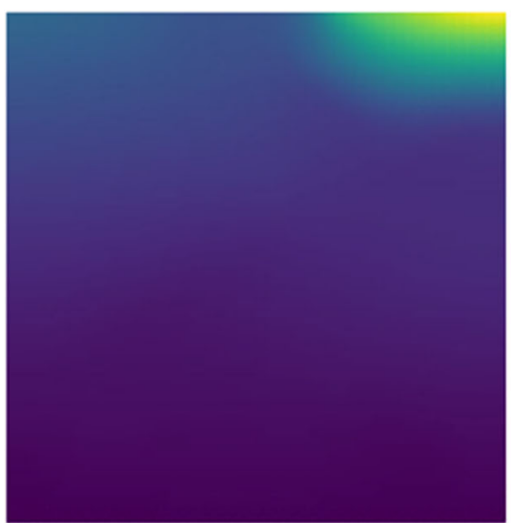

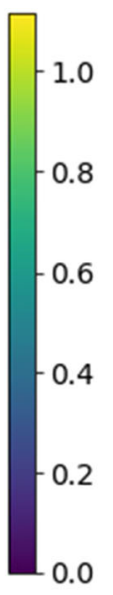

\section{Observations}

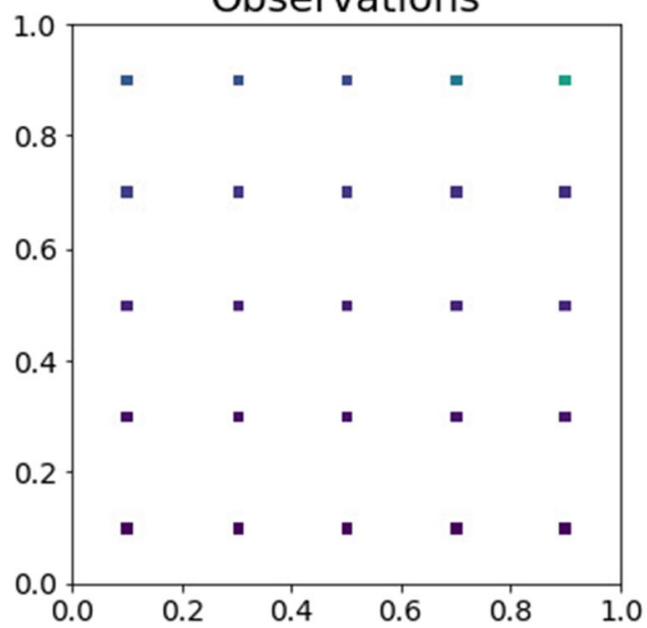

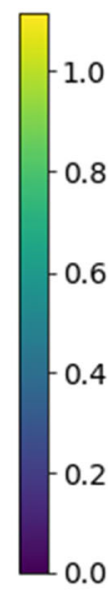

Fig. 13 The true state (left) and observation locations (right)

$\mathrm{m}$ in $\left[\begin{array}{ll}0.1 & 0.5\end{array}\right]$

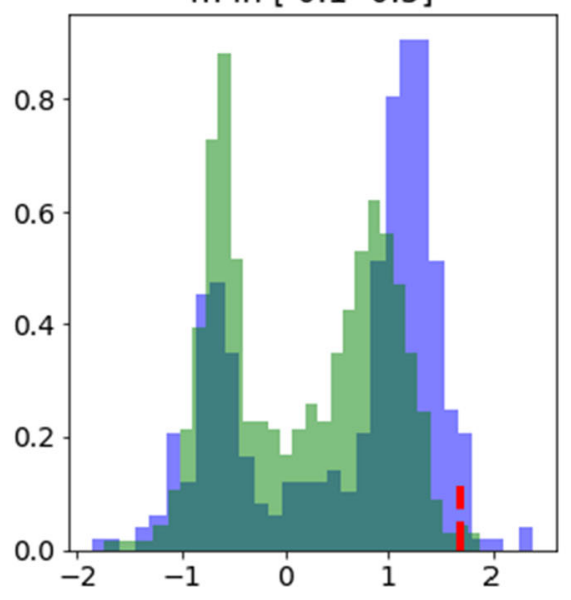

$\log K$ in $\left[\begin{array}{ll}0.1 & 0.5\end{array}\right]$

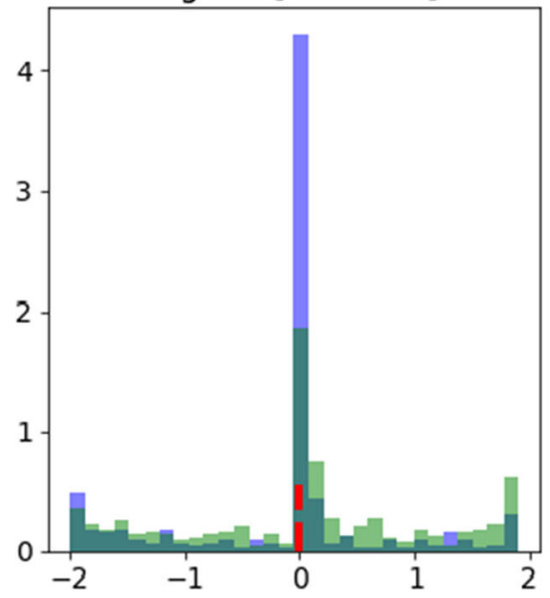

$\mathrm{m}$ in $\left[\begin{array}{ll}0.5 & 0.1\end{array}\right]$

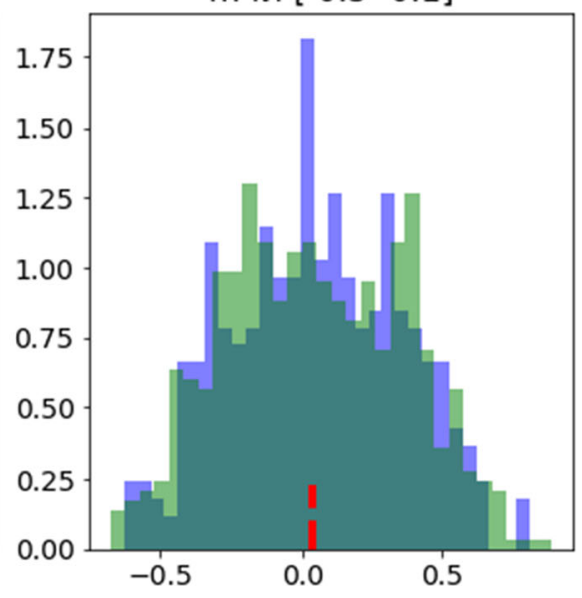

$\log K$ in $\left[\begin{array}{ll}0.5 & 0.1\end{array}\right]$

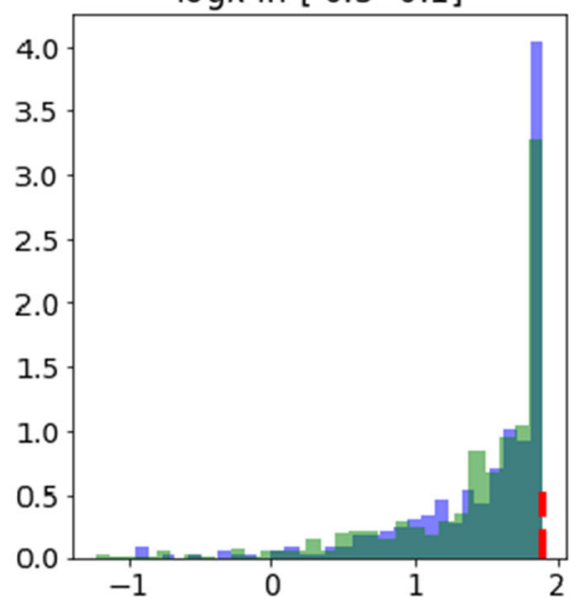

$\mathrm{m}$ in [ $\left.\begin{array}{ll}0.9 & 0.9\end{array}\right]$

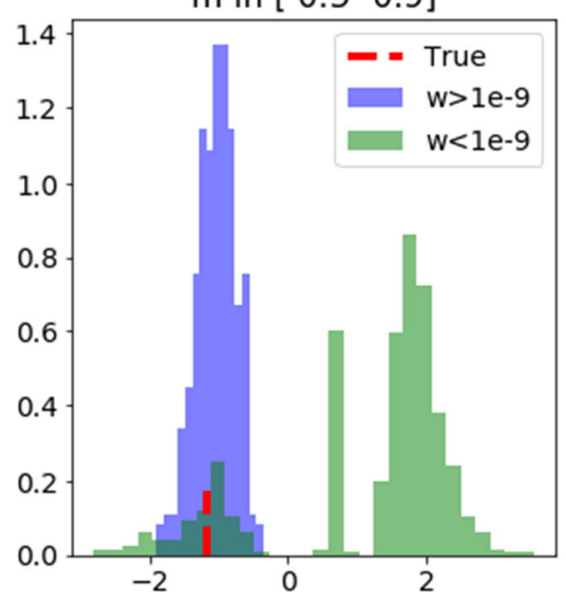

logk in [ $\left.\begin{array}{ll}0.9 & 0.9\end{array}\right]$

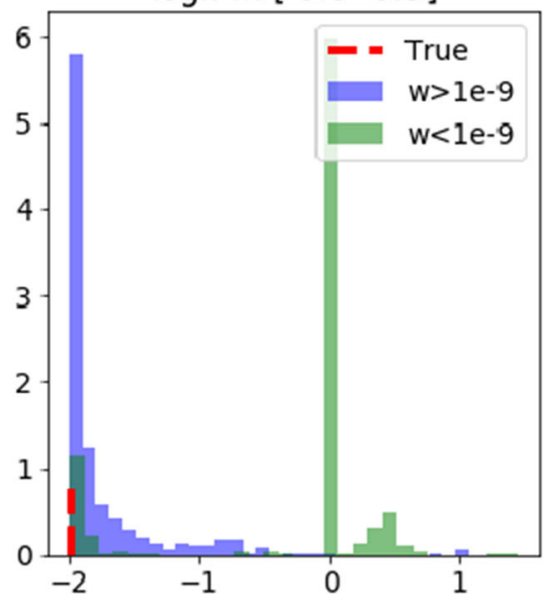

Fig. 14 Density histograms of values of the minimizers of the objective functions at three observation locations (upper row) and corresponding values of the log-perm field (lower row). Colors separate minimizers into two groups by weight 

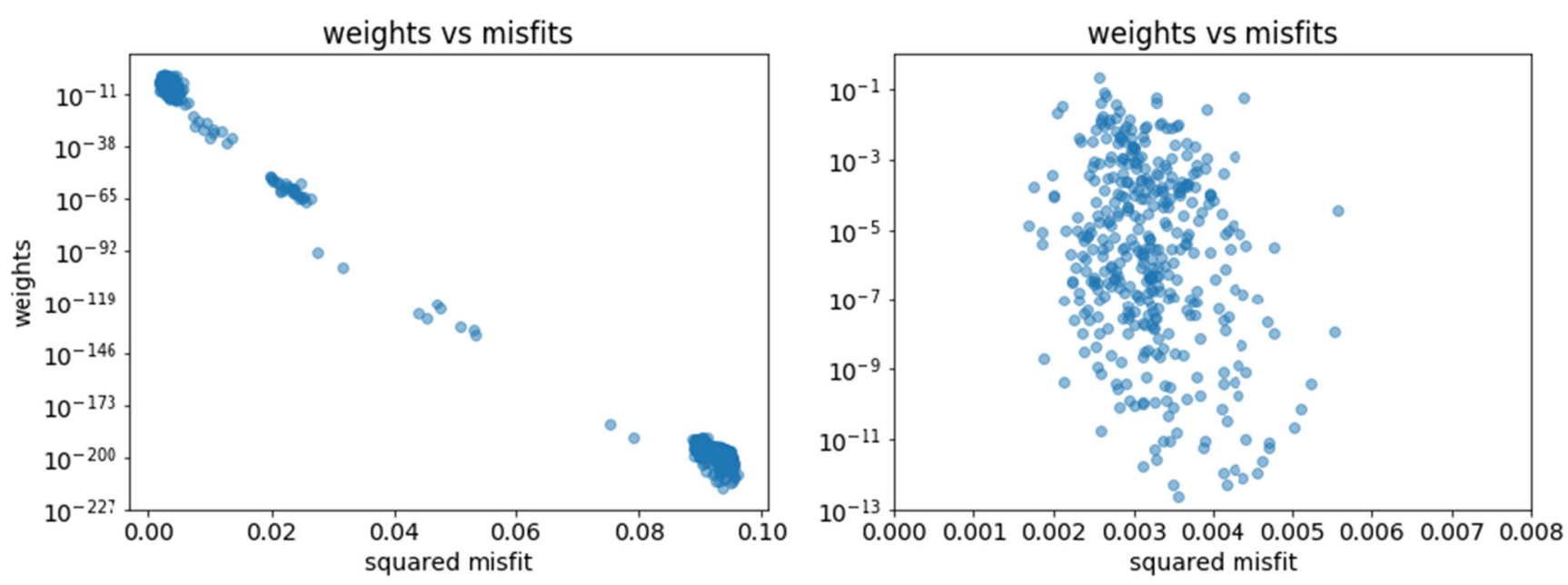

Fig. 15 The weights vs misfits for the minimizers of the stochastic cost function

the weighted RML method adequately characterizes logpermeability on the left side. It appears that it would be risky to use results from RML without weighting in this case.
As the posterior distribution is multi-modal, the mean and variance may not be the best attributes for judging the quality of the data assimilation. Two qualitative criteria are
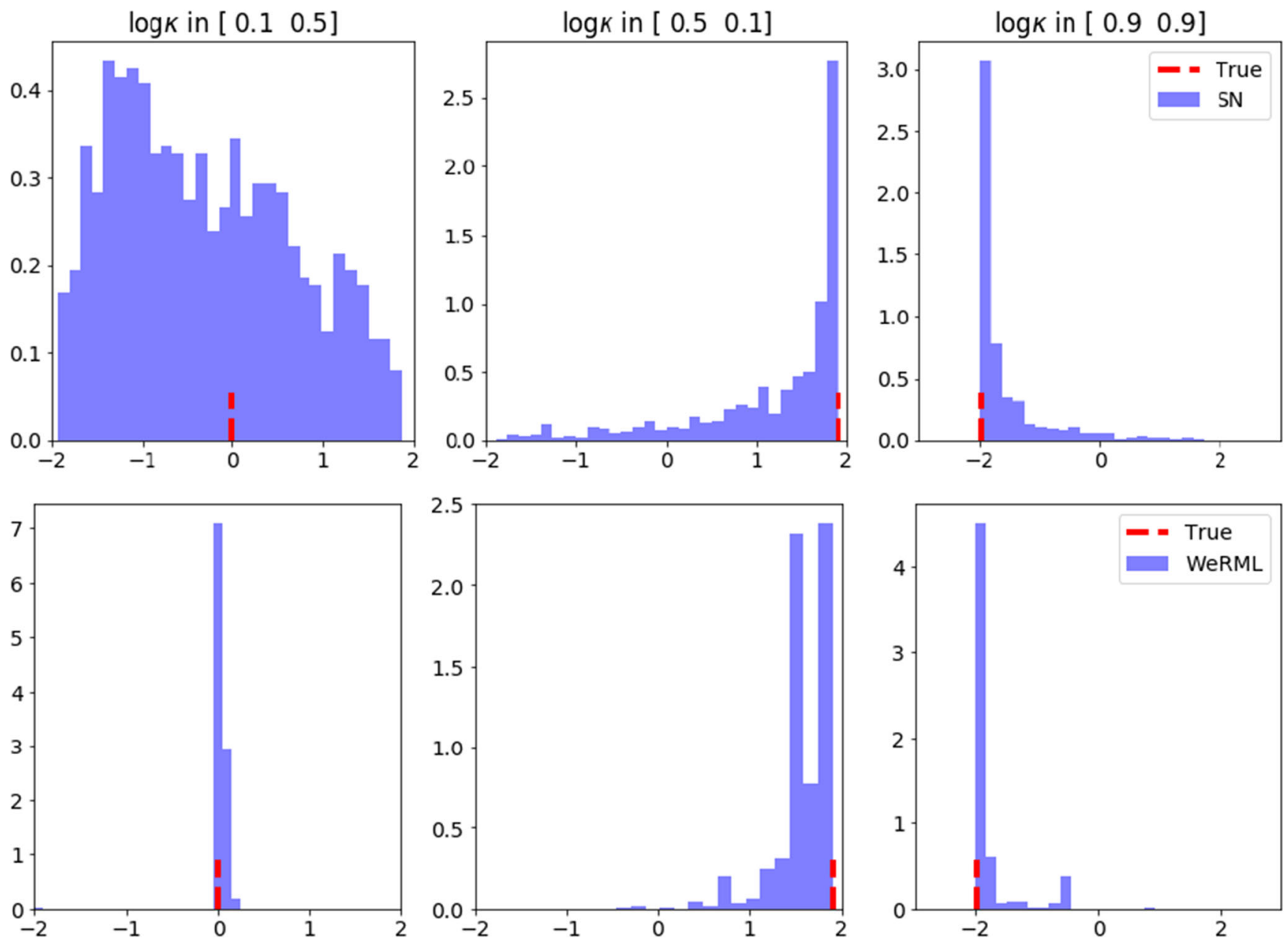

Fig. 16 The histograms of values of the log-perm field at three observation locations by using the SN (upper row) and weighted RML (lower row) methods 
often used in practice. First, it is common to judge the quality of the realizations by the data misfit after calibration. The expected value of the mean squared data mismatch with observations is 0.0025 . The value computed from weighted RML is quite close to that value, 0.0029. In contrast, the value from $\mathrm{SN}$ realizations is about 10 times larger than expected (0.0264) and the value from unweighted RML is larger still (0.0486). The distributions of squared data misfits for the three methods are shown in Fig. 12a. Second, the samples themselves can be examined qualitatively for 'plausibility' - do they look like samples from the prior? Fig. 18 shows RML samples with largest weights (bottom row) and the corresponding posterior samples for SN (top row). In this case, the weighted and unweighted RML samples look plausible, but the samples from SN do not.

\subsection{Discussion of porous flow results}

The porous flow examples were chosen to be large enough that a naïve approach to particle filtering in which particles

\section{True parameter}

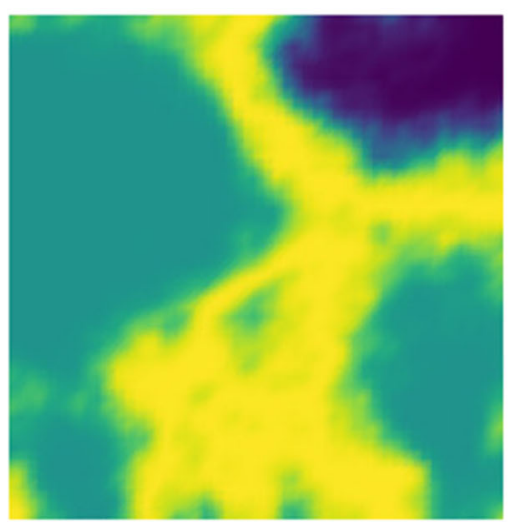

Posterior mean (RML)

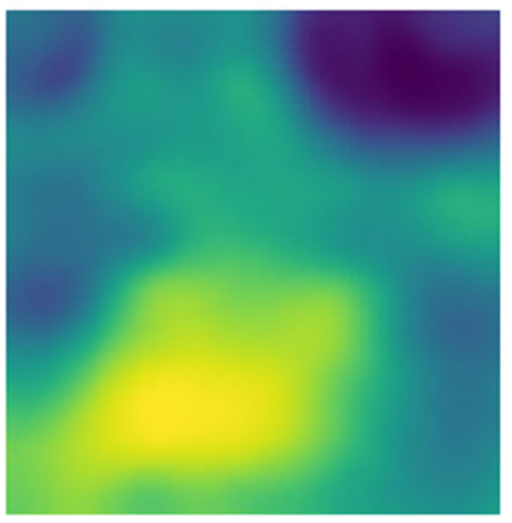

are sampled from the prior and then weighted by the likelihood would suffer from the curse of dimensionality and all the weight would fall to a single particle. The dimension of the model space (2601 discrete parameters) was also large enough that computation of the Jacobian of the transformation from the prior distribution to the distribution of critical points would be challenging.

Exact sampling of the posterior distribution using this methodology requires either computation of all critical points of the objective function, or random sampling of all critical points. In the porous media flow examples, however, we were unable to locate any maximizers for the objective function even for Case 3 in which the objective function had many local minima. As a consequence, it appears that searching only for minimizers is a robust approximation in high dimensions. Because we searched only for minimizers, it also appears that the Gauss-Newton approximation of the Jacobian gave useful approximations. The terms that must be computed are then very similar to terms that are computed in Gauss-Newton minimization of the cost

\section{Posterior mean (SN)}

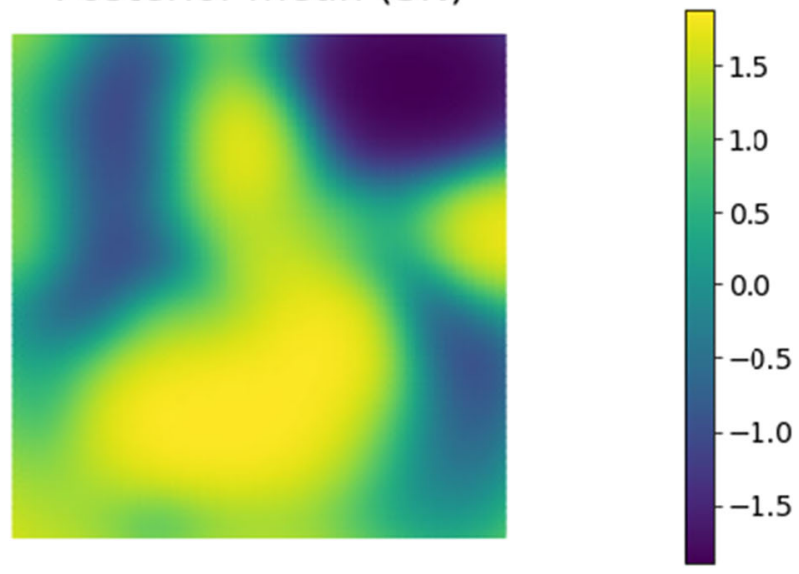

Posterior mean (WeRML)

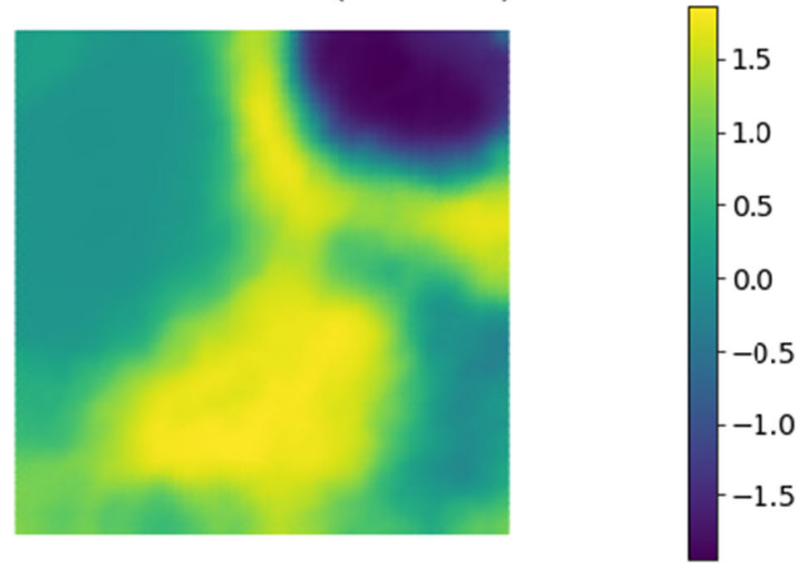

Fig. 17 The true log-permeability field for Case 3 (upper left) and the posterior mean log-permeability fields from three approximate sampling methods: stochastic Newton (upper right), RML without weighting (lower left) and RML with GN approximate weighting (lower right) 
Posterior1 (SN)
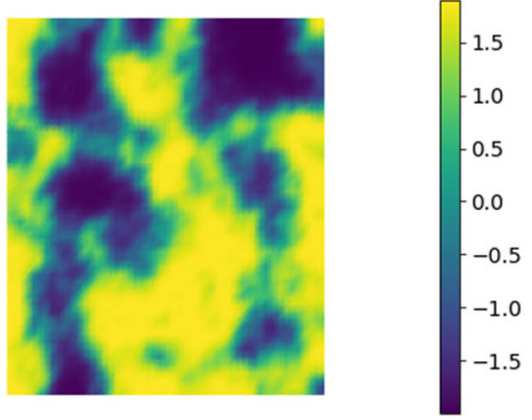

Posterior1 (RML)

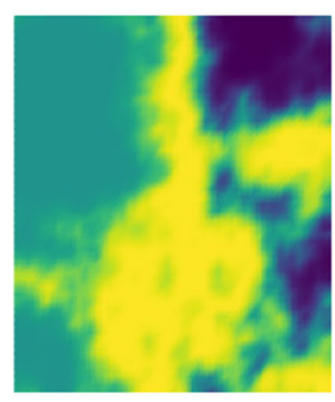

Posterior2 (SN)

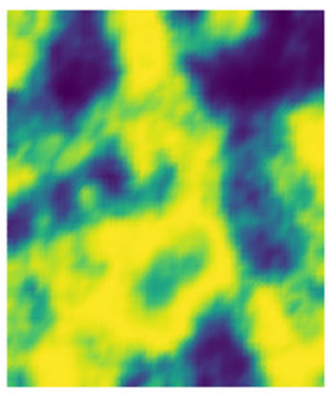

Posterior2 (RML)

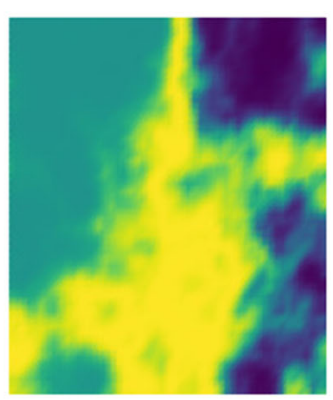

Posterior3 (SN)

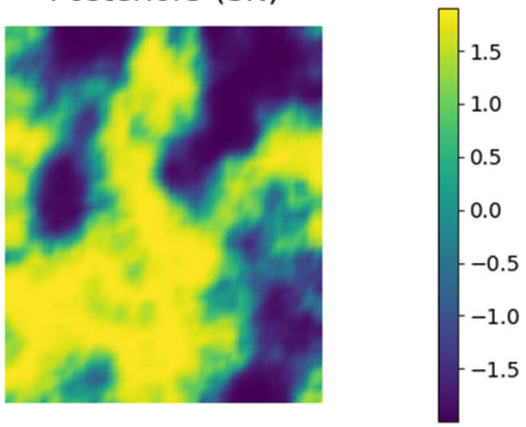

Posterior3 (RML)

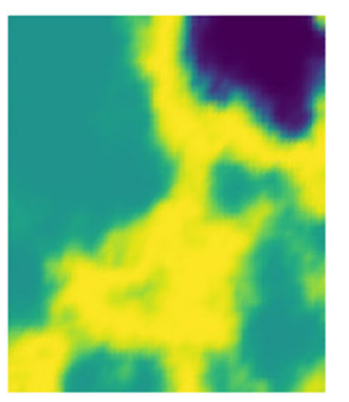

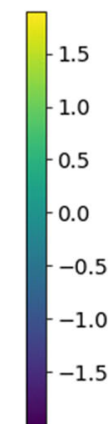

Fig. 18 Three posterior log-permeability samples from the stochastic Newton method (top row) and the three weighted RML samples with largest weights (bottom row). Samples can be compared with the true log-permeability distribution in Fig. 17

function. It was possible to compute an inexpensive estimate of the determinant of the Gauss-Newton approximation of the Jacobian using eigenvalues of the Hessian at the minimizers. Because the dimensions of the data space was relatively small, it was also possible to estimate the determinant of the Jacobian using the adjoint system. For Case 1, the estimates from the two approaches were similar, but the differences increased as the nonlinearity increased. To evaluate the quality of the sampling from the various methods, we used weights computed using the adjoint system. The weights were more variable when low rank approximations were used. In that case, it was useful to account for the model error by inflating the value of $C_{d}$ used for computing weights.

The degree of nonlinearity in the transformation from parameter to log-permeability had a strong effect on the effective efficiency of the minimization approach for sampling. The least nonlinear example (Case 1) had an effective sample efficiency of $84 \%$ while the most nonlinear example (Case 3) had an effective sample efficiency of $1.6 \%$. It appears that the low efficiency in Case 3 was largely a result of the prevalence of many local minima in the objective function, many of which were characterized by large data mismatch and very small weights.

When the posterior distribution had a single mode as in Case 1, the distribution of residual errors in the data mismatch was quite small and the correlation between weight and data mismatch was correspondingly small ( $r=$ $-0.086)$. In that case, the data mismatch would not have provided a useful proxy for weighting. In Case 2, the nonlinearity was greater but it appears that the posterior distribution was still uni-modal. The weights did correlate with data mismatch in that case $(r=-0.485)$ but it appears that the skewness of the distribution may have been the largest reason for the decrease in effective sample efficiency. Finally, in Case 3, the transformation from the model parameter to log-permeability was non-monotonic and the posterior distribution was characterized by a large number of local minima. Here, the correlation between importance weight and data mismatch was almost perfect $(r=-0.999)$ and the data mismatch could serve as a useful tool for eliminating samples with small weights.

Although we did not compare the distribution of samples from weighted RML with methods such as MCMC, we did compare with the stochastic Newton method because it is a practical and scalable method for approximate sampling in high dimensions. For Case 1, which appears to be unimodal, the mean log-permeability fields from SN and RML (both weighted and unweighted) were visually similar. For Case 3 , the mean permeability fields from SN and weighted RML are less similar, the data mismatches from SN are substantially larger, and the samples are visually less plausible.

The documented cost of the three considered methods are substantially different. The computational complexity 
of the stochastic Newton method stems from a single minimization to compute the MAP and the cost to generate samples from a low-rank Gaussian approximation of the posterior. While the sampling step contributes to the cost of the stochastic Newton, it is dominated by minimization of the objective function. For the Darcy flow example, the cost to generate 1000 approximate samples varied from 13 seconds for the log-normal case to 56 seconds for the non-monotonic case. ${ }^{3}$ Note that this increase is a result of the varying number of iterations required for the minimizer to converge for the different settings. In case of the RML method, the cost to generate $N_{e}$ realizations is dominated by the cost to perform $N_{e}$ minimizations with different cost functions. Henceforth the computational complexity for RML can be expected to be approximately $N_{e}$ times greater than the cost for stochastic Newton method. Indeed, in our examples, the run time required to generate 1000 samples from RML was approximately 1000 times greater, varying from 13000 seconds for the log-normal case to $47000 \mathrm{sec}-$ onds for the non-monotonic case. For weighted RML, there is an additional cost incurred in the computation of the weights. Although several of the terms in the weights can be obtained at low cost through the same low-rank approximations that were used for the Hessian, we chose tosolve the adjoint system $N_{d}$ times to compute the Jacobian of the data for computation of $V^{-1}$. The additional cost for computing the weighting is thus dominated by the cost of running the simulator an additional $N_{d}$ times for each realization. Because the adjoint system was solved to compute weights, the cost of computing weights varied from 24000 seconds to 35000 seconds for 1000 samples, which was similar to the cost of the minimization. All computational costs, including the cost of minimization, could be reduced through careful modification of the algorithms. In particular, the efficiency of the weighted RML could be improved by tempering the objective function at early iterations to avoid convergence to local minima with small weights. Also, the cost of computation of the weights could be reduced by using a low-rank approximation of $V$ as in the ensemble Kalman filter.

\section{Summary}

We have presented a method for sampling from the posterior distribution for inverse problems in which the prior distribution of model variables and measurements errors are Gaussian. Although the method is highly efficient when the posterior distribution is also approximately Gaussian, the target application is to problems in which the

\footnotetext{
${ }^{3}$ Timing should be considered illustrative, but for reference all results were obtained on a computer with a i7-5500U@2.40GHz $\times 4$ processor with $7.5 \mathrm{GiB}$ memory and a 64-bit operating system.
}

posterior distribution is multimodal - situations in which Gaussian approximations of the posterior distribution are inappropriate. Because of the requirement that the prior distribution be Gaussian, this method then is probably more appropriate for parameter estimation problems than for state estimation problems in which the prior may be multimodal as a result of nonlinear dynamics [31]. The method is similar to the method of randomized maximum likelihood or randomized maximum a posteriori in that samples are generated from the prior distribution and then moved to regions of high probability. Instead of solving only for minimizers of a stochastic cost function, however, the method samples correctly when all critical points are sampled and weighted, or when the critical points are randomly sampled and weighted. This procedure sampled correctly in small multimodal and skewed toy problems for which it was possible to compute all critical points. In those cases, it was also possible to obtain good approximate sampling using only minimizers of the cost function and a relatively inexpensive Gauss-Newton approximation of the particle weights.

The toy problems showed that the weights on maximizers are generally small and cannot be computed accurately using the Gauss-Newton approximation. Consequently a practical approach for larger inverse problems is to compute only the minimizers of the cost function and use approximate weights. We showed that the weights can be computed from low-rank approximations of the Hessian evaluated at the minimizers. This approach was applied to three porous media flow examples. In the first example the posterior pdf appears to be unimodal. In that case, the spread in the weights was small and the effective sample efficiency was $84 \%$. The distribution was not visibly different from samples obtained using a less expensive low rank approximation of the Hessian, but the quality of the match to the data was significantly better.

The flow example with a non-monotonic transformation to log-permeability was much more difficult. In this case, the cost function was characterized by a large number of local minimizers with small probability mass. Many of the local minimizers could be easily rejected on the basis of either low weights or unexpectedly large mismatch with observations. Because of the difficulty of converging to minimizers with large weights, the variance of the weights was large and the effective sampling efficiency in this case was low, approximately $1.6 \%$. We emphasize, however, that this example was chosen to be extremely nonlinear to test the ability to sample the multimodal posterior distribution for a moderately large model with thousands of parameters. The weighted mean of the samples from RML in this case, provided a good approximation to the true permeability distribution and the weighted data mismatches were close to the expected value. 


\section{Appendix}

Table 1 Notation used throughout the manuscript

\begin{tabular}{|c|c|c|c|}
\hline \multicolumn{4}{|c|}{ List of notations } \\
\hline$N_{m}$ & number of model parameters & $\mathrm{N}(\cdot, \cdot)$ & Gaussian distribution \\
\hline$N_{d}$ & dimension of observational space & $C_{M}$ & covariance matrix of Gaussian prior \\
\hline$N_{e}$ & number of samples & $C_{D}$ & covariance matrix of Gaussian observation error \\
\hline$N_{s}$ & number of samples of prior & $p_{M \Delta}(m, \delta)$ & target density \\
\hline$N_{\text {Eff }}$ & effective sample size & $w$ & weights \\
\hline$d^{\mathrm{o}}$ & observations & $\bar{m}$ & mean of Gaussian prior \\
\hline$m_{i}$ & ith sample of posterior distribution & $\epsilon$ & observation error \\
\hline$g$ & forward map & $\pi_{D}\left(d^{\mathrm{o}}\right)$ & normalisation constant \\
\hline$L(m)$ & log likelihood function & $\pi_{M}\left(m \mid d^{\mathrm{o}}\right)$ & posterior distribution \\
\hline$q_{M^{\prime} \Delta^{\prime}}\left(m^{\prime}, \delta^{\prime}\right)$ & proposal distribution & $\Psi$ & map between samples \\
\hline $\mathcal{M}_{z^{\prime}}$ & set mapping to $z^{\prime}$ via $\Psi$ & $n\left(z^{\prime}\right)$ & cardinality of $\mathcal{M}_{z^{\prime}}$ \\
\hline$A_{0}, A_{1}, A_{2}$ & normalisation constants & $\kappa(x)$ & permeability field \\
\hline$J$ & Jacobian determinant & $m^{*}$ & unknown reference parameter \\
\hline$m$ and $\delta$ & samples of target distribution & $m^{\prime}$ and $\delta^{\prime}$ & samples of Gaussian $q_{M^{\prime} \Delta^{\prime}}\left(m^{\prime}, \delta^{\prime}\right)$ \\
\hline$z$ & tuple of samples $m$ and $\delta$ & $m_{\mathrm{MAP}}$ & optimizer of cost functional \\
\hline$z^{\prime}$ & tuple of samples $m^{\prime}$ and $\delta^{\prime}$ & $V, \eta(m)$ & auxiliary variables \\
\hline$\sigma_{d}$ & standard deviation of measurement variables & $\sigma_{m}$ & standard deviation of parameter variables \\
\hline$u(x)$ & pressure & $\kappa^{\text {true }}$ & reference permeability field \\
\hline$n\left(m^{\prime}\right)$ & total number of critical points & G & differential operator of $g$ \\
\hline$H_{\text {map }}, H_{\text {misfit }}$ & Hessian matrices & $\Lambda$ & diagonal matrix of eigenvalues \\
\hline$Q$ & square root of $C_{M}$ & $\lambda_{i}$ & eigenvalues \\
\hline
\end{tabular}

Funding Open Access funding provided by NORCE Norwegian Research Centre AS. For this work, Yuming Ba was supported by the China Scholarship Council. Dean Oliver was supported by the NORCE Norwegian Research Centre cooperative research project "Assimilating 4D Seismic Data: Big Data Into Big Models" which is funded by industry partners Aker BP, Equinor, Lundin Norway, Repsol, and Total, as well as the Research Council of Norway through the Petromaks 2 program. Jana de Wiljes and Sebastian Reich have been partially funded by Deutsche Forschungsgemeinschaft (DFG) Project-ID 318763901 - SFB1294.

Data Availability No data are used in the manuscript.

Code Availability Selected Python codes used in the preparation of this manuscript are available at https://bitbucket.org/JanadeWiljes/ workspace/projects/RBPS.

\section{Declarations}

Conflict of Interests The authors declare that there are no conflicts of interest.

Open Access This article is licensed under a Creative Commons Attribution 4.0 International License, which permits use, sharing, adaptation, distribution and reproduction in any medium or format, as long as you give appropriate credit to the original author(s) and the source, provide a link to the Creative Commons licence, and indicate if changes were made. The images or other third party material in this article are included in the article's Creative Commons licence, unless indicated otherwise in a credit line to the material. If material is not included in the article's Creative Commons licence and your intended use is not permitted by statutory regulation or exceeds the permitted use, you will need to obtain permission directly from the copyright holder. To view a copy of this licence, visit http://creativecommons. org/licenses/by/4.0/.

\section{References}

1. Abhyankar, S., Brown, J., Constantinescu, E.M., Ghosh, D., Smith, B.F., Zhang, H.: PETSc/TS: A modern scalable ODE/DAE solver library. arXiv:1806.01437 (2018)

2. Armstrong, M., Galli, A., Beucher, H., Le Lonh, G., Renard, D., Doligez, B., Eschard, R., Geffroy, F. Plurigaussian Simulations in Geosciences, 2nd edn. Springer, Berlin (2011)

3. Bardsley, J., Solonen, A., Haario, H., Laine, M.: Randomizethen-optimize: A method for sampling from posterior distributions in nonlinear inverse problems. SIAM J. Sci. Comput. 36(4), A1895-A1910 (2014)

4. Bardsley, J.M., Cui, T., Marzouk, Y.M., Wang, Z.: Scalable optimization-based sampling on function space. SIAM J. Sci. Comput. 42(2), A1317-A1347 (2020). https://doi.org/10.1137/ $19 \mathrm{~m} 1245220$ 
5. Bengtsson, T., Bickel, P., Li, B.: Curse-Of-Dimensionality Revisited: Collapse of the Particle Filter in Very Large Scale Systems. In: Probability and Statistics: Essays in Honor of David A. Freedman, pp. 316-334. Institute of Mathematical Statistics (2008)

6. Beskos, A., Crisan, D., Jasra, A.: On the stability of sequential Monte Carlo methods in high dimensions. Ann. Appl. Probab. 24(4), 1396-1445 (2014)

7. Bjarkason, E.K., O'Sullivan, J.P., Yeh, A., O’Sullivan, M.J.: Inverse modeling of the natural state of geothermal reservoirs using adjoint and direct methods. Geothermics 78, 85-100 (2019). https://doi.org/10.1016/j.geothermics.2018.10.001

8. Bui-Thanh, T., Ghattas, O., Martin, J., Stadler, G.: A computational framework for infinite-dimensional bayesian inverse problems part i: The linearized case, with application to global seismic inversion. SIAM J. Sci. Comput. 35(6), A2494-A2523 (2013)

9. Cardiff, M., Barrash, W., Kitanidis, P.K.: A field proof-of-concept of aquifer imaging using 3-D transient hydraulic tomography with modular, temporarily-emplaced equipment. Water Resour. Res. 48(5), W05531 (2012). https://doi.org/10.1029/2011WR011704

10. Chen, Y., Oliver, D.S.: Ensemble randomized maximum likelihood method as an iterative ensemble smoother. Math. Geosci. 44(1), 1-26 (2012). https://doi.org/10.1007/s11004-011-9376-z

11. Cui, T., Law, K.J.H., Marzouk, Y.M.: Dimension-independent likelihood-informed MCMC. J. Comput. Phys. 304, 109-137 (2016). https://doi.org/10.1016/j.jcp.2015.10.008

12. Duncan, A.B., Lelièvre, T., Pavliotis, G.A.: Variance reduction using nonreversible Langevin samplers. J. Stat. Phys. 163(3), 457-491 (2016)

13. Efendiev, Y., Hou, T., Luo, W.: Preconditioning Markov chain Monte Carlo simulations using coarse-scale models. SIAM J. Sci. Comput. 28(2), 776-803 (2006)

14. Eydinov, D., Gao, G., Li, G., Reynolds, A.C.: Simultaneous estimation of relative permeability and porosity/permeability fields by history matching production data. J. Can Petrol Technol. 48(12), 13-25 (2009)

15. Freeze, R.A.: A stochastic-conceptual analysis of one-dimensional groundwater flow in a non-uniform, homogeneous media. Water Resour. Res. 11(5), 725-741 (1975)

16. Gao, G., Zafari, M., Reynolds, A.C.: Quantifying uncertainty for the PUNQ-s3 problem in a Bayesian setting with RML and enKF. SPE J. 11(4), 506-515 (2006)

17. Gelman, A.: Inference and Monitoring Convergence. In: Gilks, W.R., Richardson, S., Spiegelhalter, D.J. (eds.) Markov Chain Monte Carlo in Practice, chap. 8, pp. 131-144. Chapman \& Hall (1996)

18. Haario, H., Saksman, E., Tamminen, J.: Adaptive proposal distribution for random walk Metropolis algorithm. Comput. Stat. 14(3), 375-396 (1999)

19. Haario, H., Saksman, E., Tamminen, J.: An adaptive Metropolis algorithm. Bernoulli 7(2), 223-242 (2001)

20. Jardak, M., Talagrand, O.: Ensemble variational assimilation as a probabilistic estimator - part 2: The fully non-linear case. Nonlinear Processes Geophys. 25(3), 589-604 (2018)

21. Kitanidis, P.K.: Quasi-linear geostatistical theory for inversing. Water Resour. Res. 31(10), 2411-2419 (1995)

22. Langtangen, H.P., Logg, A.: Solving PDEs in Python: The FEniCS Tutorial I, vol. 1. Springer, Berlin (2016)

23. Law, K.J.H., Stuart, A.M.: Evaluating data assimilation algorithms. Mon. Weather. Rev. 140(11), 3757-3782 (2012). https://doi.org/10.1175/MWR-D-11-00257.1

24. van Leeuwen, P.J., Künsch, H.R., Nerger, L., Potthast, R., Reich, S.: Particle filters for high-dimensional geoscience applications: a review. Q. J. Roy. Meteorol. Soc. 145(723), 2335-2365 (2019). https://doi.org/10.1002/qj.3551
25. Liu, N., Oliver, D.S.: Evaluation of Monte Carlo methods for assessing uncertainty. SPE J. 8(2), 188-195 (2003). https://doi.org/10.2118/84936-PA

26. Liu, N., Oliver, D.S.: Automatic history matching of geologic facies. SPE J. 9(4), 188-195 (2004). https://doi.org/10.2118/ 84594-PA

27. Logg, A., Mardal, K.A., Wells, G.: Automated solution of differential equations by the finite element method: The FEniCS book, vol. 84. Springer Science \& Business Media, Berlin (2012)

28. MacKay, D.J.C.: Information theory, inference and learning algorithms. Cambridge University Press, Cambridge (2003)

29. Martin, J., Wilcox, L., Burstedde, C., Ghattas, O.: A stochastic Newton MCMC method for large-scale statistical inverse problems with application to seismic inversion. SIAM J. Sci. Comput. 34(3), A1460-A1487 (2012)

30. Martino, L., Elvira, V., Luengo, D., Corander, J.: MCMC-Driven Adaptive Multiple Importance Sampling. In: Interdisciplinary Bayesian Statistics, pp. 97-109. Springer, Berlin (2015)

31. Morzfeld, M., Hodyss, D.: Gaussian approximations in filters and smoothers for data assimilation. Tellus A: Dynamic Meteorology and Oceanography 71(1), 1-27 (2019). https://doi.org/10.1080/ 16000870.2019.1600344

32. Oliver, D.S.: Metropolized randomized maximum likelihood for improved sampling from multimodal distributions. SIAM/ASA J. Uncertain. Quantif. 5(1), 259-277 (2017). https://doi.org/10.1137/ 15M1033320

33. Oliver, D.S., Chen, Y.: Recent progress on reservoir history matching: a review. Comput. Geosci. 15(1), 185-221 (2011). https://doi.org/10.1007/s10596-010-9194-2

34. Oliver, D.S., Chen, Y.: Data assimilation in truncated plurigaussian models: impact of the truncation map. Math. Geosci. 50(8), 867-893 (2018). https://doi.org/10.1007/s11004-018-9753-y

35. Oliver, D.S., Cunha, L.B., Reynolds, A.C.: Markov chain Monte Carlo methods for conditioning a permeability field to pressure data. Math. Geol. 29(1), 61-91 (1997). https://doi.org/10.1007/BF 02769620

36. Oliver, D.S., He, N., Reynolds, A.C.: Conditioning permeability fields to pressure data. In: Proceedings of the European Conference on the Mathematics of Oil Recovery, V, pp. 1-11 (1996)

37. Roberts, G.O., Rosenthal, J.S.: Examples of adaptive MCMC. J. Comput. Graph. Stat. 18(2), 349-367 (2009)

38. Sakov, P., Oliver, D.S., Bertino, L.: An iterative enKF for strongly nonlinear systems. Mon. Weather Rev. 140(6), 1988-2004 (2012)

39. Tarantola, A.: Inverse problem theory and methods for model parameter estimation society for industrial and applied mathematics (2005)

40. Tavassoli, Z., Carter, J.N., King, P.R.: An analysis of history matching errors. Comput. Geosci. 9(2), 99-123 (2005)

41. Villa, U., Petra, N., Ghattas, O.: hIPPYlib: An extensible software framework for large-scale inverse problems. J. Open Source Softw. 3(30), 940 (2018)

42. Villa, U., Petra, N., Ghattas, O.: HIPPYLib: An extensible software framework for large-scale inverse problems governed by PDEs: Part i: Deterministic inversion and linearized Bayesian inference. ACM Trans. Math. Softw 47(2). https://doi.org/10.1145/3428447 (2021)

43. Vrugt, J.A., Nualláin, B.Ó.., Robinson, B.A., Bouten, W., Dekker, S.C., Sloot, P.M.: Application of parallel computing to stochastic parameter estimation in environmental models. Comput. Geosci. 32(8), 1139-1155 (2006)

44. Wang, K., Bui-Thanh, T., Ghattas, O.: A randomized maximum a posteriori method for posterior sampling of high dimensional nonlinear Bayesian inverse problems. SIAM J. Sci. Comput. 40(1), A142-A171 (2018) 
45. White, J.T.: A model-independent iterative ensemble smoother for efficient history-matching and uncertainty quantification in very high dimensions. Environmental Modelling \& Software (2018)

46. Zhang, F., Reynolds, A.C., Oliver, D.S.: The impact of upscaling errors on conditioning a stochastic channel to pressure data. SPE J. 8(1), 13-21 (2003)
47. Zhang, H., Constantinescu, E.M., Smith, B.F.: PETSc TSAdjoint: A discrete adjoint ODE solver for first-order and second-order sensitivity analysis. arXiv:1912.07696 (2019)

Publisher's note Springer Nature remains neutral with regard to jurisdictional claims in published maps and institutional affiliations. 